TRANSACTIONS OF THE

AMERICAN MATHEMATICAL SOCIETY

Volume 365, Number 3, March 2013, Pages 1125-1147

S 0002-9947(2012)05464-X

Article electronically published on November 6, 2012

\title{
A CALDERO-CHAPOTON MAP FOR INFINITE CLUSTERS
}

\author{
PETER JØRGENSEN AND YANN PALU
}

ABSTRACT. We construct a Caldero-Chapoton map on a triangulated category with a cluster tilting subcategory which may have infinitely many indecomposable objects.

The map is not necessarily defined on all objects of the triangulated category, but we show that it is a (weak) cluster map in the sense of Buan-IyamaReiten-Scott. As a corollary, it induces a surjection from the set of exceptional objects which can be reached from the cluster tilting subcategory to the set of cluster variables of an associated cluster algebra.

Along the way, we study the interaction between Calabi-Yau reduction, cluster structures, and the Caldero-Chapoton map.

We apply our results to the cluster category $\mathscr{D}$ of Dynkin type $A_{\infty}$ which has a rich supply of cluster tilting subcategories with infinitely many indecomposable objects. We show an example of a cluster map which cannot be extended to all of $\mathscr{D}$.

The case of $\mathscr{D}$ also permits us to illuminate results by Assem-ReutenauerSmith on $\mathrm{SL}_{2}$-tilings of the plane.

\section{INTRODUCTION}

The Caldero-Chapoton map was introduced in [10. It formalises the connection between the cluster algebras of Fomin-Zelevinsky [15] and the cluster categories of Buan-Marsh-Reineke-Reiten-Todorov 7 .

Cluster algebras are commutative rings with connections to areas as diverse as Calabi-Yau algebras, integrable systems, Poisson geometry, and quiver representations; see [28]. Cluster categories are, loosely speaking, certain categories of representations of finite dimensional algebras which were introduced to "categorify" cluster algebras (see the surveys [18, 19] and the references given there). Indeed, using the Caldero-Chapoton map, Caldero and Keller [13] established a bijection between the indecomposable rigid objects of a cluster category and the cluster variables of the corresponding cluster algebra (see also the appendix of [9]).

The Caldero-Chapoton map was generalized in 24] to 2-Calabi-Yau triangulated categories with cluster tilting objects. However, it is also interesting to consider categories which have no cluster tilting objects but only cluster tilting subcategories. In this situation, the cluster tilting subcategories have infinitely many indecomposable objects, so one cannot take the direct sum and thereby obtain cluster tilting objects; see for instance [6] and [21. A relevant example of this is the cluster

Received by the editors May 4, 2010 and, in revised form, September 8, 2010.

2010 Mathematics Subject Classification. Primary 13F60, 16G10, 16G20, 16G70, 18 E30.

Key words and phrases. Calabi-Yau reduction, cluster category, cluster map, cluster structure, cluster tilting subcategory, coindex, Dynkin type $A_{\infty}$, exchange pair, exchange triangle, FominZelevinsky mutation, Grassmannian, index, $K$-theory, $\mathrm{SL}_{2}$-tiling.

(C)2012 American Mathematical Society 
category $\mathscr{D}$ of Dynkin type $A_{\infty}$, studied in [16], which has no cluster tilting objects, but does have a rich supply of cluster tilting subcategories.

In this paper, we show that the methods of 24] apply to 2-Calabi-Yau triangulated categories with cluster tilting subcategories. Let $k$ be an algebraically closed field, let $\mathscr{C}$ be a $k$-linear Hom-finite Krull-Schmidt triangulated category which is 2-Calabi-Yau, and let $\mathscr{T}$ be a cluster tilting subcategory [21.

In Section 1 we define a Caldero-Chapoton map associated with $\mathscr{T}$. In this context, the Caldero-Chapoton map may not be defined on all the objects of the category $\mathscr{C}$. It is therefore not a cluster character in the sense of [24].

Nonetheless, in Section 2 we prove that it is a (weak) cluster map in the sense of [6] (Theorem 2.3). As a corollary, it induces a surjection from the set of reachable exceptional objects in $\mathscr{C}$ to the set of cluster variables of an associated cluster algebra.

In Section 6, for the particular case of the category $\mathscr{D}$, this yields an explicit example of a cluster map in the sense of [6] which cannot be nicely extended to a cluster character (Theorem 6.10).

Sections 3 through 5 are devoted to Calabi-Yau reductions. After recalling the definition and some results from [17, we describe the connection between modules over cluster tilting subcategories of $\mathscr{C}$ and its Calabi-Yau reductions. We also study the interactions between Calabi-Yau reduction, cluster structures, and the Caldero-Chapoton map. There are two purposes to this: First, the results are used for the category $\mathscr{D}$ to show positivity of its cluster maps; this is what makes some of them impossible to extend to cluster characters. Secondly, the results are used in Section 5 to prove an independent result: If the cluster tilting subcategory $\mathscr{T}$ is locally bounded, then the formulae for the Caldero-Chapoton map and for the cluster character of a well-chosen Calabi-Yau reduction coincide.

The final Section 7 has an application of our results to the study of $\mathrm{SL}_{2}$-tilings as introduced by Assem, Reutenauer, and Smith in [3].

\section{The Caldero-Chapoton formula}

In this section, we define a version of the Caldero-Chapoton map for cluster tilting subcategories with infinitely many indecomposable objects. Before doing so, we will review the necessary ingredients to make sure that everything works as needed in this situation.

Setup 1.1. Let $k$ be an algebraically closed field.

In Sections 1 1 through 5, the following notation is used:

$\mathscr{C}$ is a $k$-linear Hom-finite Krull-Schmidt triangulated category which is 2-CalabiYau. Its shift functor is $\Sigma$.

$\mathscr{T}$ is a cluster tilting subcategory of $\mathscr{C}$ and $T=$ ind $\mathscr{T}$ is its set of (isomorphism classes of) indecomposable objects. Note that $\mathscr{T}=\operatorname{add} T$.

1.2 (Modules over $\mathscr{T}$ ). Following [21, the category Mod $\mathscr{T}$ of $\mathscr{T}$-modules is the category of $k$-linear contravariant functors from $\mathscr{T}$ to the category of $k$-vector spaces. A $\mathscr{T}$-module $M$ is said to be finitely presented if it admits a presentation by representable functors:

$$
\mathscr{T}\left(-, t_{1}\right) \rightarrow \mathscr{T}\left(-, t_{0}\right) \rightarrow M \rightarrow 0 .
$$


In particular, finitely presented modules are functors from $\mathscr{T}$ to the category of finite dimensional $k$-vector spaces. The full subcategory of finitely presented modules is denoted by mod $\mathscr{T}$ and is an abelian category (see [21, 2.1]).

By [8, thm. 2.2], [21, 2.1, prop.], [22, cor. 4.4] the functor

$$
\begin{aligned}
\mathscr{C} & \stackrel{G}{\longrightarrow} \bmod \mathscr{T}, \\
c & \left.\longmapsto \mathscr{C}(-, \Sigma c)\right|_{\mathscr{T}}
\end{aligned}
$$

induces an equivalence of categories $\mathscr{C} /[\mathscr{T}] \simeq \bmod \mathscr{T}$. The square brackets denote the ideal of morphisms which factor through an object in $\mathscr{T}$.

1.3 (The results of 24). A number of results were established in 24] under the conditions of Setup 1.1, with the additional assumption that the set $T$ was finite. This assumption is unnecessary for many of the results. To wit, lemmas 3.1, 3.3, 4.2 and proposition 4.3 of 24] certainly remain true under Setup 1.1 alone, with the same proofs.

Moreover, indices and coindices of objects in $\mathscr{C}$ were introduced in 24] and further studied in [14]. If $c$ is an object of $\mathscr{C}$, then pick distinguished triangles

$$
t_{1} \rightarrow t_{0} \rightarrow c, \quad c \rightarrow \Sigma^{2} t^{0} \rightarrow \Sigma^{2} t^{1}
$$

with $t_{i}, t^{i} \in \mathscr{T}$; cf. [21, 2.1 prop] or [22, lem. 3.2(1)]. Define the index and the coindex relative to $\mathscr{T}$ as the classes

$$
\text { ind } \mathscr{T} c=\left[t_{0}\right]-\left[t_{1}\right], \quad \text { coind } \mathscr{T} c=\left[t^{0}\right]-\left[t^{1}\right]
$$

in the split Grothendieck group $\mathrm{K}_{0}^{\text {split }}(\mathscr{T})$. Here the square brackets indicate $\mathrm{K}$ classes. The subscripts of ind $\mathscr{T}$ and coind $\mathscr{T}$ will be omitted when there is no danger of confusion. This definition differs slightly from 24] where the index and coindex took values in the usual Grothendieck group $\mathrm{K}_{0}(\bmod \mathscr{T})$. However, 24, lemma 2.1 (parts 1, 2,4) and proposition 2.2] remain true under Setup 1.1 alone, again with the same proofs.

1.4 (Finite length objects). The simple objects in Mod $\mathscr{T}$ are precisely the objects $S_{t}=\operatorname{top} \mathscr{T}(-, t)$ for $t \in T$; see [5, prop. 2.3(b)].

For $t \in T$, there exists a right almost split morphism $b \rightarrow t$ in $\mathscr{T}$, and hence $S_{t} \in \bmod \mathscr{T}$ by [5, cor. 2.6]. Let us show that $b \rightarrow t$ exists.

Set $T^{\prime}=T \backslash t$ and write $\mathscr{A}=\operatorname{add} T^{\prime}$. By [17, thm. 5.3], there exists a unique indecomposable $t^{*} \neq t$ in $\mathscr{C}$ for which $\mathscr{T}^{*}=\operatorname{add}\left(T^{\prime} \cup t^{*}\right)$ is a cluster tilting subcategory. Moreover, $\left(\mathscr{T}^{*}, \mathscr{T}\right)$ is a so-called $\mathscr{A}$-mutation pair by [17, thm. 5.3] again. One consequence is that there exists a distinguished triangle $x \rightarrow a \rightarrow t \rightarrow$ $\Sigma x$ in $\mathscr{C}$ with $x \in \mathscr{T}^{*}$ and $a \in \mathscr{A}$; see [17, def. 2.5]. If $s \neq t$ is an indecomposable object in $T$, then $s \in \mathscr{T}^{*}$ and so $\mathscr{C}(s, \Sigma x)=0$. It follows that any morphism $s \rightarrow t$ factors through $a \rightarrow t$. Now pick $n$ morphisms in $\mathscr{C}(t, t)$ which generate the radical of $\mathscr{C}(t, t)$ as a right- $\mathscr{C}(t, t)$-module. They define a morphism $t^{n} \rightarrow t$, and it is not hard to see that $a \oplus t^{n} \rightarrow t$ is right almost split.

So each $S_{t}$ is in mod $\mathscr{T}$. Conversely, let $S$ be a simple object of mod $\mathscr{T}$. The category mod $\mathscr{T}$ has enough projectives, so there is an epimorphism from a projective object, $\mathscr{T}(-, t) \rightarrow S$, where $t \in \mathscr{T}$; we can clearly assume that $t$ is indecomposable. This implies that $S_{t}$ is a quotient of $S$ in Mod $\mathscr{T}$, but both of these objects are simple objects of $\bmod \mathscr{T}$, so it follows that $S \cong S_{t}$.

Hence Mod $\mathscr{T}$ and $\bmod \mathscr{T}$ have the same simple objects, namely the $S_{t}$ for $t \in T$. 
Using that the inclusion functor $\bmod \mathscr{T} \rightarrow \operatorname{Mod} \mathscr{T}$ is exact, see [4, sec. III.2], and that mod $\mathscr{T}$ is closed under extensions in Mod $\mathscr{T}$, it follows that Mod $\mathscr{T}$ and mod $\mathscr{T}$ have the same finite length objects. We denote the category of these by fl $\mathscr{T}$.

1.5 ( $K$-theory). $\quad$ (i) It is clear that there is an isomorphism

$$
\mathrm{K}_{0}^{\text {split }}(\mathscr{T}) \cong \operatorname{Free}(T) .
$$

(ii) There is the following homomorphism:

$$
\begin{aligned}
& \mathrm{K}_{0}(\bmod \mathscr{T}) \stackrel{\theta_{\mathscr{T}}}{\longrightarrow} \mathrm{K}_{0}^{\text {split }}(\mathscr{T}), \\
& {[G c] \longmapsto \operatorname{coind}_{\mathscr{T}} \Sigma c-\operatorname{ind}_{\mathscr{T}} \Sigma c \text {, }}
\end{aligned}
$$

where the subscript of $\theta_{\mathscr{T}}$ will be omitted when there is no danger of confusion. Namely, the expression coind $\Sigma c-$ ind $\Sigma c$ depends only on $G c$ by [24, lem. 2.1(4)], and respects the relations of $\mathrm{K}_{0}(\bmod \mathscr{T})$ by [24, lem. 3.1 and prop. 2.2].

The embedding fl $\mathscr{T} \rightarrow \bmod \mathscr{T}$ induces a homomorphism $\mathrm{K}_{0}(\mathrm{fl} \mathscr{T}) \rightarrow$ $\mathrm{K}_{0}(\bmod \mathscr{T})$, which we can compose with $\theta$; by abuse of notation, the composition will also be denoted by $\theta$.

We will use this homomorphism in place of the bilinear form $\langle-,-\rangle_{a}$ of 24. If the quiver of $\mathscr{T}$ is locally finite and has no loops, then they are related via the following formula:

$$
\theta(-)=\sum_{t \in T}\left\langle S_{t},-\right\rangle_{a}[t]
$$

Note that if $\mathscr{T}$ belongs to a cluster structure on $\mathscr{C}$ in the sense of 6 , sec. II.1], then each $t \in T$ sits in so-called exchange triangles $t^{*} \rightarrow c \rightarrow t$, $t \rightarrow c^{\prime} \rightarrow t^{*}$, where $t^{*}$ is the unique replacement of $t$ which leaves $\mathscr{T}$ a cluster tilting subcategory, and where $c, c^{\prime} \in \operatorname{add}(T \backslash t)$. In that case we have $S_{t}=G t^{*}$ and a computation shows

$$
\theta\left(\left[S_{t}\right]\right)=[c]-\left[c^{\prime}\right]
$$

(iii) We introduce variables $x_{t}$ for $t \in T$. If $\alpha=\sum_{t} \alpha_{t}[t]$ is an element of $\mathrm{K}_{0}^{\text {split }}(\mathscr{T})$, then we write

$$
x^{\alpha}=\prod_{t} x_{t}^{\alpha_{t}}
$$

this is a well-defined monomial, which we view as an element of the rational function field $\mathbb{Q}\left(x_{t}\right)_{t \in T}$ generated by the $x_{t}$.

1.6 (Grassmannians). For each $t \in T$ the functor $S_{t} \in \mathrm{fl} \mathscr{T}$ is zero at each indecomposable different from $t$. If $M \in \mathfrak{f l} \mathscr{T}$ satisfies $[M]=e$ in $\mathrm{K}_{0}(\mathrm{fl} \mathscr{T})$, where $e=\sum_{t} e_{t}\left[S_{t}\right]$, then $M$ has a composition series with quotients $S_{t}$ for which $e_{t} \neq 0$. It follows that the functor $M$ is zero at each indecomposable $t$ with $e_{t}=0$. So $M$ is supported at the finitely many $t \in T$ with $e_{t} \neq 0$.

Now let $N \in \bmod \mathscr{T}$ and consider subfunctors $M \subseteq N$, where $M$ has finite length and satisfies $[M]=e$ in $\mathrm{K}_{0}(\mathrm{fl} \mathscr{T})$. Then $M$ is supported at the finitely many $t \in T$ with $e_{t} \neq 0$, so $M$ can be viewed as an $m$-dimensional sub-vector space of the finite dimensional $k$-vector space $E=\bigoplus_{\left\{t \mid e_{t} \neq 0\right\}} N(t)$, and $m$ is determined by $e$. There is a Grassmannian of $m$-dimensional subspaces of $E$, and it follows that 
there is a Grassmannian $\operatorname{Gr}_{e}(N)$ whose points parametrize the subfunctors $M \subseteq N$, where $M$ has finite length and satisfies $[M]=e$. Both Grassmannians are algebraic spaces.

It is also possible to introduce auxiliary algebraic spaces in the manner of [24, sec. 5.1]. Namely, let $\ell, m \in \mathscr{C}$ be such that $G \ell, G m$ have finite length, and suppose that there are distinguished triangles

$$
m \stackrel{i}{\rightarrow} b \stackrel{p}{\rightarrow} \ell, \quad \ell \stackrel{i^{\prime}}{\rightarrow} b^{\prime} \stackrel{p^{\prime}}{\rightarrow} m
$$

in $\mathscr{C}$. Note that $G b, G b^{\prime}$ also have finite length. Let $e, f \in \mathrm{K}_{0}(\mathrm{fl} \mathscr{T})$ and set

$$
\begin{aligned}
X_{e, f} & =\left\{E \subseteq G b \mid\left[(G i)^{-1} E\right]=e,[(G p) E]=f\right\}, \\
Y_{e, f} & =\left\{E^{\prime} \subseteq G b^{\prime} \mid\left[\left(G i^{\prime}\right)^{-1} E^{\prime}\right]=f,\left[\left(G p^{\prime}\right) E^{\prime}\right]=e\right\} .
\end{aligned}
$$

There are morphisms of algebraic spaces

$$
\begin{aligned}
& X_{e, f} \rightarrow \operatorname{Gr}_{e}(G m) \times \operatorname{Gr}_{f}(G \ell) \leftarrow Y_{e, f}, \\
& E \mapsto \quad\left((G i)^{-1} E, \quad(G p) E\right), \\
& \left(\left(G p^{\prime}\right) E^{\prime}, \quad\left(G i^{\prime}\right)^{-1} E^{\prime}\right) \longleftrightarrow E^{\prime}
\end{aligned}
$$

for which each fibre is an affine space $k^{n}$. This property of the fibres is due to [10, see their lemma 3.8, and it can be proved in the present situation by following the proof of [25, lem. 3.2] to obtain a free transitive action of the algebraic group $k^{n}$ on a fibre. In fact, for a point $E \in X_{e, f}$ one sets $V=(G p) E$ and $W=$ $\operatorname{Coker}\left((G i)^{-1} E \hookrightarrow G m\right)$, and it is $(\bmod \mathscr{T})(V, W)$, viewed as an algebraic group isomorphic to $k^{n}$, which acts on the fibre containing $E$. Note that $V$ and $W$ have finite length in $\bmod \mathscr{T}$, so $(\bmod \mathscr{T})(V, W)$ is indeed finite dimensional over $k$.

There are now two cases of interest.

Case 1: The triangles (11) are split. Then there is a (split) exact sequence $0 \rightarrow$ $G m \stackrel{G i}{\rightarrow} G b \stackrel{G p}{\rightarrow} G \ell \rightarrow 0$ which implies

$$
\operatorname{Gr}_{g}(G b)=\coprod_{e+f=g} X_{e, f} .
$$

Moreover, the morphism $X_{e, f} \rightarrow \mathrm{Gr}_{e}(G m) \times \mathrm{Gr}_{f}(G \ell)$ is surjective since $M \oplus L$ is sent to $(M, L)$. The fibres are affine spaces, so we get

$$
\chi\left(\operatorname{Gr}_{e}(G m) \times \operatorname{Gr}_{f}(G \ell)\right)=\chi\left(X_{e, f}\right),
$$

where $\chi$ is an (ordinary or $\ell$-adic) Euler characteristic.

The last two equations give the first and third equality in the following key formula:

$$
\begin{aligned}
\sum_{e+f=g} \chi\left(\operatorname{Gr}_{e}(G m) \times \operatorname{Gr}_{f}(G \ell)\right) & =\sum_{e+f=g} \chi\left(X_{e, f}\right) \\
& =\chi\left(\coprod_{e+f=g} X_{e, f}\right)=\chi\left(\operatorname{Gr}_{g}(G b)\right) .
\end{aligned}
$$

Note that the sums are finite since the relevant algebraic spaces are only non-empty for $e, f, g$ in the positive cone of $\mathrm{K}_{0}(\mathrm{fl} \mathscr{T})$.

Case 2: We have $\operatorname{Ext}_{\mathscr{C}}^{1}(\ell, m)=1$ and the triangles (1) are not split. The morphisms from $X_{e, f}$ and $Y_{e, f}$ induce a morphism from the disjoint union

$$
X_{e, f} \amalg Y_{e, f} \rightarrow \operatorname{Gr}_{e}(G m) \times \operatorname{Gr}_{f}(G \ell) .
$$


It follows from [24, prop. 4.3] that this morphism is surjective and that each point in the target is the image either of a point from $X_{e, f}$ or a point from $Y_{e, f}$, but not both. Hence the fibres are still affine spaces $k^{n}$, so

$$
\chi\left(\operatorname{Gr}_{e}(G m) \times \operatorname{Gr}_{f}(G \ell)\right)=\chi\left(X_{e, f} \amalg Y_{e, f}\right)=\chi\left(X_{e, f}\right)+\chi\left(Y_{e, f}\right) .
$$

Following [24, sec. 5.1] in setting

$$
X_{e, f}^{g}=X_{e, f} \cap \operatorname{Gr}_{g}(G b), \quad Y_{e, f}^{g}=Y_{e, f} \cap \operatorname{Gr}_{g}\left(G b^{\prime}\right)
$$

for $g \in \mathrm{K}_{0}(\mathrm{fl} \mathscr{T})$ hence gives another key formula,

$$
\chi\left(\operatorname{Gr}_{e}(G m) \times \operatorname{Gr}_{f}(G \ell)\right)=\sum_{g} \chi\left(X_{e, f}^{g}\right)+\chi\left(Y_{e, f}^{g}\right) .
$$

It is clear that

$$
\chi\left(\operatorname{Gr}_{g}(G b)\right)=\sum_{e, f} \chi\left(X_{e, f}^{g}\right), \quad \chi\left(\operatorname{Gr}_{g}\left(G b^{\prime}\right)\right)=\sum_{e, f} \chi\left(Y_{e, f}^{g}\right) .
$$

The sums in equations (3) and (4) are also finite. Namely, if $e, f$ are fixed, then each of $X_{e, f}^{g}$ and $Y_{e, f}^{g}$ is only non-empty for finitely many values of $g$. If $g$ is fixed, then each of $X_{e, f}^{g}$ and $Y_{e, f}^{g}$ is only non-empty for finitely many values of $(e, f)$.

The following is a version of [24, lem. 5.1], and a version of the proof of that result works. We stay within the situation of Paragraph 1.6 .

Lemma 1.7. If e, $f, g \in \mathrm{K}_{0}(\mathrm{fl} \mathscr{T})$ are such that $X_{e, f}^{g} \neq \emptyset$, then

$$
\theta(g)-\operatorname{coind} \Sigma b=\theta(e+f)-\operatorname{coind} \Sigma m-\operatorname{coind} \Sigma \ell .
$$

1.8 (The Caldero-Chapoton formula for cluster tilting subcategories). Let $c \in \mathscr{C}$. Using the material from the previous paragraphs, we give the following version of the Caldero-Chapoton formula from [10, sec. 3]:

$$
\rho^{\mathscr{T}}(c)=x^{-\operatorname{coind} \mathscr{T} \Sigma c} \sum_{e} \chi\left(\mathrm{Gr}_{e}(G c)\right) x^{\theta_{\mathscr{T}}(e)} .
$$

The sum is over $e$ in $\mathrm{K}_{0}(\mathfrak{f} \mid \mathscr{T})$, and the superscript of $\rho^{\mathscr{T}}$ will be omitted when there is no danger of confusion.

For an arbitrary $c$, it is not clear that $\rho(c)$ is well-defined. However, if $G c$ has finite length in $\bmod \mathscr{T}$, then $\rho(c)$ is a well-defined element of $\mathbb{Z}\left[x_{t}, x_{t}^{-1}\right]_{t \in T}$, and hence of $\mathbb{Q}\left(x_{t}\right)_{t \in T}$, because the sum is finite. Namely, since the functor $G c$ has finite length, it is only supported at finitely many $t \in T$. By the deliberations of Paragraph 1.6. this implies that there are only finitely many classes $e$ in $\mathrm{K}_{0}(\mathrm{fl} \mathscr{T})$ for which $G c$ has a submodule $M$ with $[M]=e$, and hence only finitely many classes $e$ in $\mathrm{K}_{0}(\mathrm{fl} \mathscr{T})$ for which $\mathrm{Gr}_{e}(G c) \neq \emptyset$.

The following version of [10, cor. 3.7] works under Setup 1.1] without the assumption that $T$ is finite.

Proposition 1.9. Suppose that $m, \ell \in \mathscr{C}$ have $G m, G \ell$ of finite length in mod $\mathscr{T}$. Then $\rho(m), \rho(\ell)$ are elements of $\mathbb{Z}\left[x_{t}, x_{t}^{-1}\right]_{t \in T}$ which satisfy

$$
\rho(m \oplus \ell)=\rho(m) \rho(\ell) .
$$

Proof. We have $\rho(m), \rho(\ell) \in \mathbb{Z}\left[x_{t}, x_{t}^{-1}\right]_{t \in T}$ by Paragraph 1.8, and the formula is an immediate consequence of equation (2). 
The following version of [24, thm. 1.4] also works under Setup 1.1 alone.

Proposition 1.10. Suppose that $m, \ell \in \mathscr{C}$ have $\operatorname{dim} \operatorname{Ext}_{\mathscr{C}}^{1}(m, \ell)=1$ and that $\mathrm{Gm}$, Gl have finite length in mod $\mathscr{T}$.

Let

$$
m \rightarrow b \rightarrow \ell, \quad \ell \rightarrow b^{\prime} \rightarrow m
$$

be non-split distinguished triangles in $\mathscr{C}$. Then $\rho(m), \rho(\ell), \rho(b), \rho\left(b^{\prime}\right)$ are elements of $\mathbb{Z}\left[x_{t}, x_{t}^{-1}\right]_{t \in T}$ which satisfy

$$
\rho(m) \rho(\ell)=\rho(b)+\rho\left(b^{\prime}\right) .
$$

Proof. The objects $b, b^{\prime}$ also have $G b, G b^{\prime}$ of finite length, so $\rho(m), \rho(\ell), \rho(b), \rho\left(b^{\prime}\right) \in$ $\mathbb{Z}\left[x_{t}, x_{t}^{-1}\right]_{t \in T}$ by Paragraph 1.8 .

To get the equation, we use the proof of [24, thm. 1.4] given in [24, sec. 5.1] which also works in the present situation. Only a modest computation is necessary; we show it here:

$$
\begin{aligned}
\rho(m) \rho(\ell) & =x^{-\operatorname{coind} \Sigma m} \sum_{e} \chi\left(\operatorname{Gr}_{e} G m\right) x^{\theta e} \times x^{-\operatorname{coind} \Sigma \ell} \sum_{f} \chi\left(\operatorname{Gr}_{f} G \ell\right) x^{\theta f} \\
& =x^{-\operatorname{coind} \Sigma m-\operatorname{coind} \Sigma \ell} \sum_{e, f} \chi\left(\operatorname{Gr}_{e} G m\right) \chi\left(\operatorname{Gr}_{f} G \ell\right) x^{\theta(e+f)} \\
& =x^{-\operatorname{coind} \Sigma m-\operatorname{coind} \Sigma \ell} \sum_{e, f} \chi\left(\operatorname{Gr}_{e}(G m) \times \operatorname{Gr}_{f}(G \ell)\right) x^{\theta(e+f)} \\
& \stackrel{(\mathrm{a})}{=} x^{-\operatorname{coind} \Sigma m-\operatorname{coind} \Sigma \ell} \sum_{e, f, g}\left(\chi\left(X_{e, f}^{g}\right)+\chi\left(Y_{e, f}^{g}\right)\right) x^{\theta(e+f)} \\
& \stackrel{(\mathrm{b})}{=} \sum_{e, f, g} \chi\left(X_{e, f}^{g}\right) x^{\theta(g)-\operatorname{coind} \Sigma b}+\sum_{e, f, g} \chi\left(Y_{e, f}^{g}\right) x^{\theta(g)-\operatorname{coind} \Sigma b^{\prime}} \\
& \stackrel{(\mathrm{c})}{=} x^{-\operatorname{coind} \Sigma b} \sum_{g} \chi\left(\mathrm{Gr}_{g} G b\right) x^{\theta g}+x^{-\operatorname{coind} \Sigma b^{\prime}} \sum_{g} \chi\left(\mathrm{Gr}_{g} G b^{\prime}\right) x^{\theta g} \\
& =\rho(b)+\rho\left(b^{\prime}\right) .
\end{aligned}
$$

Here (a) is by equation (3), (b) is by Lemma 1.7 and its analogue for $Y$ and $b^{\prime}$, and (c) is by equation (4).

\section{Cluster maps}

Recall that we work under Setup 1.1. In this section, we prove that the CalderoChapoton map associated with a cluster tilting subcategory is a (weak) cluster map in the sense of $[6$.

Setup 2.1. In this section we assume that the cluster tilting subcategories which can be reached by finitely many mutations from $\mathscr{T}$ form a cluster structure in the sense of [6, sec. II.1].

Note that this forces the quiver $Q_{\mathscr{T}}$ of $\mathscr{T}$ to be locally finite.

The set of indecomposable objects belonging to cluster tilting subcategories which can be reached from $\mathscr{T}$ will be denoted by $E$, and we will write $\mathscr{E}=$ add $E$.

2.2 (Cluster maps). We recall the following from [6, sec. IV.1]. A map

$$
\varphi: \operatorname{obj} \mathscr{E} \rightarrow \mathbb{Q}\left(x_{t}\right)_{t \in T}
$$


is called a cluster map if it satisfies the following conditions:

(i) $\varphi$ is constant on isomorphism classes.

(ii) If $c_{1}, c_{2} \in \mathscr{E}$, then $\varphi\left(c_{1} \oplus c_{2}\right)=\varphi\left(c_{1}\right) \varphi\left(c_{2}\right)$.

(iii) If $m, \ell \in \operatorname{obj} \mathscr{E}$ are indecomposable objects with $\operatorname{dim}_{k} \operatorname{Ext}_{\mathscr{C}}^{1}(m, \ell)=1$ and $b, b^{\prime} \in \operatorname{obj} \mathscr{E}$ are such that there are non-split distinguished triangles

$$
m \rightarrow b \rightarrow \ell, \quad \ell \rightarrow b^{\prime} \rightarrow m
$$

in $\mathscr{C}$, then $\varphi(m) \varphi(\ell)=\varphi(b)+\varphi\left(b^{\prime}\right)$.

(iv) There is a cluster tilting subcategory $\mathscr{T}^{\prime}$ which can be reached from $\mathscr{T}$ for which $\left\{\varphi\left(t^{\prime}\right) \mid t^{\prime} \in\right.$ ind $\left.\mathscr{T}^{\prime}\right\}$ is a transcendence basis of the field $\mathbb{Q}\left(x_{t}\right)_{t \in T}$.

Theorem 2.3. The Caldero-Chapoton formula for cluster tilting subcategories gives a well-defined cluster map

$$
\rho: \operatorname{obj} \mathscr{E} \rightarrow \mathbb{Q}\left(x_{t}\right)_{t \in T}
$$

satisfying $\rho(t)=x_{t}$ for each $t \in T$.

Proof. Let us first show that $G(\varepsilon)$ has finite length for $\varepsilon \in \operatorname{obj} \mathscr{E}$. It is enough to show that $G(e)$ has finite length when $e$ is an indecomposable object belonging to a cluster tilting subcategory $\mathscr{T}^{\prime}$ which can be obtained from $\mathscr{T}$ by successive mutations at finitely many objects $t_{1}, \ldots, t_{m}$. But ind $\mathscr{T}^{\prime}$ is equal to $T \backslash\left\{t_{1}, \ldots, t_{m}\right\}$ united with finitely many indecomposable objects, and it follows that $\mathscr{C}(t, \Sigma e)=0$ for $t \in T \backslash\left\{t_{1}, \ldots, t_{m}\right\}$. Hence $G(e)$ is supported on $\left\{t_{1}, \ldots, t_{m}\right\}$ and so it has finite length.

Let us now prove the theorem. The equality $\rho(t)=x_{t}$ for $t \in T$ is shown by a direct computation. Paragraph 1.8 combined with the finite length of $G(\varepsilon)$ shows that $\rho$ is well-defined on obj $\mathscr{E}$ and takes values in $\mathbb{Z}\left[x_{t}, x_{t}^{-1}\right]_{t \in T}$ and hence in $\mathbb{Q}\left(x_{t}\right)_{t \in T}$. Property (i) of cluster maps is clear, property (ii) holds by Proposition 1.9. and property (iii) by Proposition 1.10 .

Property (iv) holds with $\mathscr{T}^{\prime}=\mathscr{T}$ since $\rho(t)=x_{t}$.

2.4 (Cluster algebras). Recall that cluster algebras were originally introduced by Fomin and Zelevinsky in [15. We will not recall their definition, but merely remind the reader that a cluster algebra is generated by cluster variables which form overlapping sets of fixed cardinality called clusters.

The clusters in 15] were finite, but cluster algebras with countable clusters are defined in [6, sec. IV.1].

Corollary 2.5. Suppose that $\mathscr{T}$ has countably many indecomposable objects and let $\mathscr{A}$ be the cluster algebra associated with the quiver $Q_{\mathscr{T}}$.

The map $\rho$ induces a surjection from $E$ to the cluster variables of $\mathscr{A}$. Under the surjection, the indecomposable objects of a cluster tilting subcategory which can be reached from $\mathscr{T}$ are sent to a cluster.

Proof. Immediate from Theorem 2.3 and [6, thm. IV.1.2(a)].

\section{Calabi-Yau Reductions}

The technique of Calabi-Yau reductions, introduced by Iyama and Yoshino in [17, sec. 4], yields fully faithful functors between the module categories of different cluster tilting subcategories. In this section, we recall the construction of CalabiYau reductions and give a nice description of these functors. 
Setup 3.1. In this section we let $U \subseteq T$ be a subset for which $\mathscr{U}=\operatorname{add} U$ is functorially finite in $\mathscr{C}$.

For a subcategory $\mathscr{V}$ of $\mathscr{C}$, we let $\perp^{\perp \mathscr{V}}$ (resp. $\mathscr{V}^{\perp}$ ) denote the full subcategory of $\mathscr{C}$ whose objects are left (resp. right) Hom-orthogonal to each object of $\mathscr{V}$.

3.2 (Calabi-Yau reductions). We recall the following from [17, sec. 4]. Set

$$
\mathscr{C}_{U}={ }^{\perp}(\Sigma \mathscr{U}) /[\mathscr{U}]
$$

and note that $\mathscr{C}_{\emptyset}=\mathscr{C}$ and $\mathscr{C}_{T}=0$. The category $\mathscr{C}_{U}$ is triangulated. Its suspension functor $\Sigma_{U}$ is defined on objects as follows: Let $c \in{ }^{\perp}(\Sigma \mathscr{U})$ and let $c \rightarrow u_{c}$ be a minimal left- $\mathscr{U}$-approximation. Complete it to a distinguished triangle in $\mathscr{C}$,

$$
c \rightarrow u_{c} \rightarrow \Sigma_{U} c \stackrel{\sigma_{c}}{\rightarrow} \Sigma c,
$$

where $\Sigma_{U} c$ belongs to ${ }^{\perp}(\Sigma \mathscr{U})$ by the Wakamatsu lemma. Let $c \rightarrow d \rightarrow e \rightarrow \Sigma c$ be a distinguished triangle in $\mathscr{C}$, with $c, d, e \in{ }^{\perp}(\Sigma \mathscr{U})$. Since $e$ belongs to ${ }^{\perp}(\Sigma \mathscr{U})$, the composition $e \rightarrow \Sigma c \rightarrow \Sigma u_{c}$ vanishes and the identity of $c$ induces a morphism of triangles

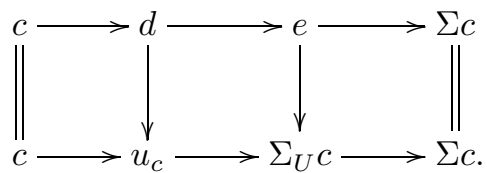

The image in $\mathscr{C}_{U}$ of the complex $c \rightarrow d \rightarrow e \rightarrow \Sigma_{U} c$ is a distinguished triangle, and all distinguished triangles in $\mathscr{C}_{U}$ are isomorphic to such.

For $c, d \in{ }^{\perp}(\Sigma \mathscr{U})$, applying the functor $\mathscr{C}(d,-)$ to the triangle (5) shows that composition by $\sigma_{c}$ induces an isomorphism

$$
\mathscr{C}_{U}\left(d, \Sigma_{U} c\right) \stackrel{\simeq}{\longrightarrow} \mathscr{C}(d, \Sigma c) .
$$

Moreover, $\mathscr{C}_{U}$ is a $k$-linear Hom-finite Krull-Schmidt 2-Calabi-Yau category. The projection functor ${ }^{\perp}(\Sigma \mathscr{U}) \stackrel{\pi}{\rightarrow} \mathscr{C}_{U}$ induces a bijection between the cluster tilting subcategories of $\mathscr{C}$ containing $\mathscr{U}$ and the cluster tilting subcategories of $\mathscr{C}_{U}$, and the bijection is compatible with mutation at indecomposable objects outside $\mathscr{U}$.

Example 3.3. For each $n$, the cluster category of type $A_{n}$ is a Calabi-Yau reduction of the cluster category $\mathscr{D}$ of type $A_{\infty}$. See Lemma 6.5 for more details on this situation.

3.4 (Modules over cluster tilting subcategories). The following formula contains an equivalence of categories which comes from the third isomorphism theorem and a fully faithful functor which is obvious:

$$
\frac{\perp(\Sigma \mathscr{U}) /[\mathscr{U}]}{[\mathscr{T} /[\mathscr{U}]]} \simeq \frac{\perp(\Sigma \mathscr{U})}{[\mathscr{T}]} \hookrightarrow \frac{\mathscr{C}}{[\mathscr{T}]}
$$

Abbreviating $\mathscr{T} /[\mathscr{U}]$ to $\mathscr{T} / \mathscr{U}$ gives a fully faithful functor $\mathscr{C}_{U} /[\mathscr{T} / \mathscr{U}] \hookrightarrow \mathscr{C} /[\mathscr{T}]$. By way of the functor $G$ of Paragraph 1.2 and its analogue $G_{U}$ for the cluster tilting subcategory $\mathscr{T} / \mathscr{U}$ of $\mathscr{C}_{U}$, this translates into a fully faithful functor

$$
\bmod \mathscr{T} / \mathscr{U} \stackrel{\pi^{*}}{\hookrightarrow} \bmod \mathscr{T}
$$

which sends $\left.\mathscr{C}_{U}\left(-, \Sigma_{U} c\right)\right|_{\mathscr{T} / \mathscr{U}}$ to $\mathscr{C}(-, \Sigma c) \mid \mathscr{T}$. 
The situation is depicted in the following diagram:

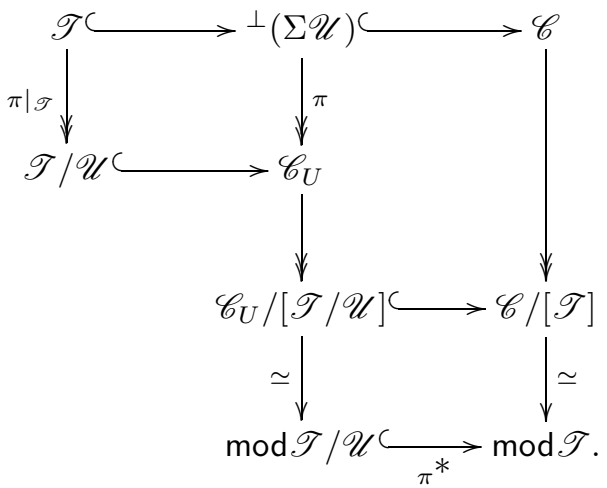

The isomorphism (6) of Paragraph 3.2 shows that the functor $\pi^{*}$ is indeed equivalent to the one induced by composition with $\pi$ : For $c \in{ }^{\perp}(\Sigma \mathscr{U})$ the $\mathscr{T}$-modules $\left.\mathscr{C}_{U}\left(-, \Sigma_{U} c\right)\right|_{\mathscr{T} / \mathscr{U}} \circ \pi \mid \mathscr{T}$ and $\left.\mathscr{C}(-, \Sigma c)\right|_{\mathscr{T}}$ are isomorphic.

Proposition 3.5. $\quad$ (i) The fully faithful functor $\pi^{*}$ is exact.

(ii) The essential image of $\pi^{*}$ is the $\mathscr{T}$-modules vanishing on $\mathscr{U}$, so $\pi^{*}$ identifies finitely presented $\mathscr{T} / \mathscr{U}$-modules with finitely presented $\mathscr{T}$-modules vanishing on $\mathscr{U}$.

(iii) Given $M \in \bmod \mathscr{T} / \mathscr{U}$, the functor $\pi^{*}$ induces a bijection between isomorphism classes of submodules of $M$ in $\bmod \mathscr{T} / \mathscr{U}$ and isomorphism classes of submodules of $\pi^{*} M$ in $\bmod \mathscr{T}$.

Proof. (i) An exact sequence in $\bmod \mathscr{T} / \mathscr{U}$ has the form

$$
\left.\left.\left.\mathscr{C}_{U}\left(-, \Sigma_{U} c\right)\right|_{\mathscr{T} / \mathscr{U}} \rightarrow \mathscr{C}_{U}\left(-, \Sigma_{U} d\right)\right|_{\mathscr{T} / \mathscr{U}} \rightarrow \mathscr{C}_{U}\left(-, \Sigma_{U} e\right)\right|_{\mathscr{T} / \mathscr{U}}
$$

for a distinguished triangle $c \rightarrow d \rightarrow e$ in $\mathscr{C}_{U}$ (see [24, lem. 3.1]) which may be taken to come from a distinguished triangle in $\mathscr{C}$ with entries in ${ }^{\perp}(\Sigma \mathscr{U})$. But $\pi^{*}$ sends (77) to $\left.\left.\left.\mathscr{C}(-, \Sigma c)\right|_{\mathscr{T}} \rightarrow \mathscr{C}(-, \Sigma d)\right|_{\mathscr{T}} \rightarrow \mathscr{C}(-, \Sigma e)\right|_{\mathscr{T}}$, which is also exact.

(ii) Let $c \in \mathscr{C}$. By the 2-Calabi-Yau property, $\mathscr{C}(-, \Sigma c) \mid \mathscr{T}$ vanishes on $\mathscr{U}$ if and only if $c$ belongs to ${ }^{\perp}(\Sigma \mathscr{U})$.

(iii) Since $\pi^{*} M$ is a $\mathscr{T}$-module vanishing on $\mathscr{U}$, all its $\mathscr{T}$-submodules also vanish on $\mathscr{U}$.

\section{Calabi-Yau Reductions and the Caldero-Chapoton map}

This section shows an aspect of the interaction between Calabi-Yau reductions and the Caldero-Chapoton map.

Lemma 4.1. Let $R \subseteq T$ be such that $T \backslash R$ is finite. Then $\mathscr{R}=\operatorname{add} R$ is functorially finite in $\mathscr{C}$.

Remark. The proof we will give of the lemma works under weaker assumptions than the standing Setup 1.1. Namely, it is only needed that $\mathscr{C}$ has a Serre functor (not that it is 2-Calabi-Yau) and that $\mathscr{T}$ is $n$-cluster tilting for some $n$ (not that it is cluster tilting).

Proof. We only prove that $\mathscr{R}$ is contravariantly finite. Covariant finiteness then follows by duality. Since $\mathscr{T}$ is contravariantly finite in $\mathscr{C}$, it is enough to prove 
that each $t \in T \backslash R$ has a right- $\mathscr{R}$-approximation. By propositions 2.10 and 2.11 of [17, any object in $\mathscr{T}$ has a sink map. Let $b_{0} \oplus c_{0} \stackrel{a_{0}}{\longrightarrow} t$ be a sink map in $\mathscr{T}$ with $b_{0} \in \operatorname{add}(T \backslash R)$ and $c_{0} \in$ add $R$. If $b_{0}$ is zero, then $a_{0}$ is the approximation we are looking for. Otherwise, let $b_{1} \oplus c_{1} \stackrel{a_{1}}{\longrightarrow} b_{0}$ be a sink map in $\mathscr{T}$, with the same conventions as above. We then inductively construct sink maps $b_{j} \oplus c_{j} \stackrel{a_{j}}{\longrightarrow} b_{j-1}$, with the convention that $a_{j}$ is zero if $b_{j-1}$ is zero. Since $\mathscr{C}$ is Hom-finite and $T \backslash R$ is finite, there is some $m$ for which the induced composition $b_{m} \rightarrow \cdots \rightarrow b_{1} \rightarrow b_{0} \rightarrow t$ vanishes. One easily checks that $\bigoplus_{j=0}^{m} c_{j} \rightarrow t$ is a right- $\mathscr{R}$-approximation.

Lemma 4.2. Assume that the cluster tilting subcategories which can be reached from $\mathscr{T}$ form a cluster structure in $\mathscr{C}$, in the sense of [6, sec. II.1]. Then, for any subset $U \subseteq T$ such that $\mathscr{U}=$ add $U$ is functorially finite, the cluster tilting subcategories which can be reached from $\mathscr{T} / \mathscr{U}$ form a cluster structure in the Calabi-Yau reduction $\mathscr{C}_{U}$.

Proof. By [6, thm. II.1.6], it is enough to check that the cluster tilting subcategories reachable from $\mathscr{T} / \mathscr{U}$ in $\mathscr{C}_{U}$ have no loops or 2-cycles. Moreover, since the CalabiYau reduction is compatible with mutation, it is enough to check the following: If $\mathscr{T}$ has no loops or 2 -cycles in $\mathscr{C}$, then $\mathscr{T} / \mathscr{U}$ has no loops or 2 -cycles in $\mathscr{C}_{U}$. This follows easily from the fact that the exchange triangles in $\mathscr{C}_{U}$ are the images of the exchange triangles in $\mathscr{C}$.

Setup 4.3. In the rest of this section, we let $U \subseteq T$ be a subset for which $\mathscr{U}=$ add $U$ is functorially finite in $\mathscr{C}$, and we assume that the cluster tilting subcategories which can be reached from $\mathscr{T} / \mathscr{U}$ form a cluster structure in $\mathscr{C}_{U}$.

Note that hence, the results of Sections 1 and 3 apply in $\mathscr{C}$ and the results of Section 2 apply in $\mathscr{C}_{U}$.

Notation 4.4. The set of indecomposable objects belonging to cluster tilting subcategories of $\mathscr{C}_{U}$ which can be reached from $\mathscr{T} / \mathscr{U}$ will be denoted by $F$, and we will write $\mathscr{F}=\operatorname{add} F$.

Remark 4.5. Objects in $\mathscr{C}_{U}$ are identified with the objects of $\perp(\Sigma \mathscr{U}) \subseteq \mathscr{C}$ without direct summands in $\mathscr{U}$. Under this identification, the objects in $\mathscr{C}$ corresponding to objects in $\mathscr{F}$ are sent to finite length modules by the functor $G$; indeed, they are sent to finite length $\mathscr{T} / \mathscr{U}$-modules by the proof of Theorem 2.3. The following definition therefore makes sense:

Definition 4.6. Let the map

$$
\bar{\rho}^{\mathscr{T}}:\left.\operatorname{obj} \mathscr{F} \rightarrow \mathbb{Q}\left(x_{t}\right)\right|_{t \in T \backslash U}
$$

be given by $\bar{\rho}^{\mathscr{T}}(f)=\left.\rho^{\mathscr{T}}(f)\right|_{x_{u}=1 \text { for } u \in U}$.

Proposition 4.7. The map $\bar{\rho}^{\mathscr{T}}$ : obj $\left.\mathscr{F} \rightarrow \mathbb{Q}\left(x_{t}\right)\right|_{t \in T \backslash U}$ is a cluster map.

Proof. We must prove that the map $\bar{\rho}^{\mathscr{T}}$ satisfies the conditions of Paragraph 2.2 . Conditions (i) and (ii) follow immediately from the corresponding properties of $\rho^{\mathscr{T}}$.

To see (iii), let $m, \ell \in \mathscr{F}$ be indecomposable objects with $\operatorname{dim}_{k} \operatorname{Ext}_{\mathscr{C}_{U}}^{1}(m, \ell)=1$ and suppose that there are $b, b^{\prime} \in \mathscr{F}$ and non-split distinguished triangles

$$
m \rightarrow b \rightarrow \ell, \quad \ell \rightarrow b^{\prime} \rightarrow m
$$


in $\mathscr{C}_{U}$. By the isomorphism (6) of Paragraph 3.2. we get $\operatorname{dim}_{k} \operatorname{Ext}_{\mathscr{C}}^{1}(m, \ell)=1$ and so the two triangles are isomorphic in $\mathscr{C}_{U}$ to the images of distinguished triangles in $\mathscr{C}$ :

$$
m \rightarrow b \oplus u \rightarrow \ell, \quad \ell \rightarrow b^{\prime} \oplus u^{\prime} \rightarrow m
$$

where $u, u^{\prime} \in \mathscr{U}$. By Remark 4.5 we have that $G m, G \ell$ have finite length, so Propositions 1.9 and 1.10 give

$$
\rho^{\mathscr{T}}(m) \rho^{\mathscr{T}}(\ell)=\rho^{\mathscr{T}}(b \oplus u)+\rho^{\mathscr{T}}\left(b^{\prime} \oplus u^{\prime}\right)=\rho^{\mathscr{T}}(b) \rho^{\mathscr{T}}(u)+\rho^{\mathscr{T}}\left(b^{\prime}\right) \rho^{\mathscr{T}}\left(u^{\prime}\right),
$$

whence

$$
\bar{\rho}^{\mathscr{T}}(m) \bar{\rho}^{\mathscr{T}}(\ell)=\bar{\rho}^{\mathscr{T}}(b)+\bar{\rho}^{\mathscr{T}}\left(b^{\prime}\right) .
$$

Finally, (iv) follows as in the proof of Theorem 2.3 since $\bar{\rho}^{\mathscr{T}}(t)=x_{t}$ for each $t \in T \backslash U$.

Theorem 4.8. The maps $\rho^{\mathscr{T} / \mathscr{U}}$ and $\bar{\rho}^{\mathscr{T}}$ coincide on obj $\mathscr{F}$.

Proof. Both are cluster maps by Theorem 2.3 and Proposition 4.7. Therefore it is enough to check that they coincide on the indecomposable objects of $\mathscr{T} / \mathscr{U}$; cf. 24, Lemma 5.3]. But if $t \in T \backslash U$, then $\rho^{\mathscr{T} / \mathscr{U}}$ and $\bar{\rho}^{\mathscr{T}}$ both take the value $x_{t}$ on $t$.

Corollary 4.9. Assume that the cluster tilting subcategories which can be reached from $\mathscr{T}$ form a cluster structure in $\mathscr{C}$. Then the map $\rho^{\mathscr{T}}$ is "pointwise finite" in the following sense: For each $\ell \in$ obj $\mathscr{E}$, there exists a cluster tilting object $R$ in a Calabi-Yau reduction of $\mathscr{C}$ such that $\rho^{\mathscr{T}}(\ell)=X_{\ell}^{R}$, where $X^{R}$ is the cluster character of 24$]$.

Proof. We may take $\ell \in$ obj $\mathscr{E}$ to be indecomposable, and it sits in some cluster tilting subcategory obtained from $\mathscr{T}$ by a finite number of mutations. Therefore the spaces $\mathscr{C}(t, \Sigma \ell)$ vanish for all but finitely many $t_{1}, \ldots, t_{r} \in T$. There exist $t_{r+1}, \ldots, t_{m} \in T$ such that $\rho^{\mathscr{T}}(\ell)$ belongs to $\left.\mathbb{Q}\left(x_{t_{1}}, \ldots, x_{t_{m}}\right) \subset \mathbb{Q}\left(x_{t}\right)\right|_{t \in T}$. Let $R$ be the direct sum $t_{1} \oplus \cdots \oplus t_{m}$ and let $U$ be $T \backslash\left\{t_{1}, \ldots, t_{m}\right\}$. Lemma 4.1 ensures that the subcategory $\mathscr{U}=\operatorname{add} U$ is functorially finite. By Lemma 4.2, the cluster tilting object $R$ defines a cluster structure on the Calabi-Yau reduction $\mathscr{C}_{U}$. We can now apply Theorem 4.8 to get the desired result.

Remark 4.10. P-G. Plamondon recently defined, in [26], a Caldero-Chapoton map associated with any rigid object (in a not necessarily Hom-finite triangulated category) and proved that it behaves like a cluster map. Assume that the rigid object is a direct summand of a cluster tilting object. It would be interesting to adapt the methods used above in order to relate the Caldero-Chapoton map of [26] with the one on the Calabi-Yau reduction in which the rigid object becomes cluster tilting.

\section{Calabi-Yau Reductions in the locally bounded Case}

In the previous section, Theorem 4.8 showed a degree of compatibility between Calabi-Yau reductions and the Caldero-Chapoton map. This section goes further under the additional assumption that the cluster tilting subcategory is locally bounded. Following [23], a full subcategory $\mathscr{V}$ of $\mathscr{C}$ is called locally bounded if, for each indecomposable object $v$ in $\mathscr{V}$, there are only finitely many (isomorphism classes of) indecomposable objects $w$ in $\mathscr{V}$ such that $\mathscr{C}(v, w) \neq 0$ or $\mathscr{C}(w, v) \neq 0$.

Setup 5.1. In this section we let $U \subseteq T$ be a subset for which $\mathscr{U}=\operatorname{add} U$ is functorially finite in $\mathscr{C}$. (See Lemma 4.1 for examples of such subsets.) 
Let

$$
\kappa: K_{0}^{\text {split }}(\mathscr{T} / \mathscr{U}) \hookrightarrow K_{0}^{\text {split }}(\mathscr{T})
$$

be the canonical inclusion.

Lemma 5.2. Let $c \in{ }^{\perp}(\Sigma \mathscr{U})$; in particular, $c$ can be viewed as an object of $\mathscr{C}_{U}$ as well as of $\mathscr{C}$.

(i) If $c \in \perp \mathscr{U}$, then ind $\mathscr{T} \Sigma c=\kappa\left(\operatorname{ind}_{\mathscr{T} / \mathscr{U}} \Sigma_{U} c\right)$.

(ii) If $c \in{ }^{\perp}\left(\Sigma^{2} \mathscr{U}\right)$, then coind $\mathscr{T} \Sigma c=\kappa\left(\operatorname{coind}_{\mathscr{T}} / \mathscr{U} \Sigma_{U} c\right)$.

Proof. (i) In the distinguished triangle $c \rightarrow u_{c} \rightarrow \Sigma_{U} c \rightarrow \Sigma c$ we have $u_{c}=0$ since $c \in \perp \mathscr{U}$, so $\Sigma_{U} c$ and $\Sigma c$ are isomorphic in $\mathscr{C}_{U}$. Pick a distinguished triangle $t_{1} \rightarrow t_{0} \rightarrow \Sigma c \rightarrow \Sigma t_{1}$ with $t_{i} \in \mathscr{T}$ and $t_{0} \rightarrow \Sigma c$ right-minimal; note that $\Sigma c \rightarrow \Sigma t_{1}$ is then left-minimal. Since $c \in{ }^{\perp}(\Sigma \mathscr{U})$ it follows that $\Sigma c \in \mathscr{U}^{\perp}$ because $\mathscr{C}$ is 2-Calabi-Yau, and $c \in \perp^{\perp} \mathscr{U}$, so $\Sigma c \in{ }^{\perp}(\Sigma \mathscr{U})$. Therefore $t_{0}$ and $t_{1}$ have no direct summands in $\mathscr{U}$.

But $t_{0}, t_{1}, \Sigma c$ belong to ${ }^{\perp}(\Sigma \mathscr{U})$, so there is an induced distinguished triangle $t_{1} \rightarrow t_{0} \rightarrow \Sigma c \rightarrow \Sigma_{U} t_{1}$ in $\mathscr{C}_{U}$ which, by the beginning of the proof, is $t_{1} \rightarrow t_{0} \rightarrow$ $\Sigma_{U} c \rightarrow \Sigma_{U} t_{1}$. Since the objects $t_{i}$ have no direct summands in $\mathscr{U}$, the morphism $\kappa$ sends $\left[t_{0}\right]-\left[t_{1}\right]$ in $K_{0}^{\text {split }}(\mathscr{T} / \mathscr{U})$ to $\left[t_{0}\right]-\left[t_{1}\right]$ in $K_{0}^{\text {split }}(\mathscr{T})$.

(ii) This is dual to (i) because the coindex of $\Sigma c$ in $(\mathscr{C}, \mathscr{T})$ is the index of $\Sigma^{-1} c$ in $\left(\mathscr{C}^{\mathrm{op}}, \mathscr{T}^{\mathrm{op}}\right), \Sigma^{-1}$ is the shift functor in $\mathscr{C}^{\mathrm{op}}$, and ${ }^{\perp}\left(\Sigma^{2} \mathscr{U}\right)=\mathscr{U}^{\perp}$ by the 2-Calabi-Yau property.

Setup 5.3. In the rest of this section, we assume that the cluster tilting subcategory $\mathscr{T}$ is locally bounded.

Remark 5.4. Let $c \in \mathscr{C}$. There is a right- $\mathscr{T}$-approximation $t_{0} \rightarrow c$ and a left- $\mathscr{T}$ approximation $c \rightarrow t^{0}$. Since $\mathscr{T}$ is locally bounded, this implies that there are only finitely many indecomposable objects $t \in T$ such that $\mathscr{C}(t, c) \neq 0$ or $\mathscr{C}(c, t) \neq 0$.

This implies that the functor $G c=\left.\mathscr{C}(-, \Sigma c)\right|_{\mathscr{T}}$ has finite length in $\bmod \mathscr{T}$. By Paragraph 1.8 the Caldero-Chapoton map $\rho^{\mathscr{T}}$ is defined on all the objects of $\mathscr{C}$, whence $\rho^{\mathscr{T}}$ is a cluster character 24 . It also means that any subset $U \subseteq T$ can be used in Setup 5.1 because $\mathscr{U}=$ add $U$ is always functorially finite in $\mathscr{C}$.

Now let $s_{1}, \ldots, s_{n} \in \mathscr{C}$, without non-zero direct summands in $\mathscr{T}$, be such that the modules $\left.\mathscr{C}\left(-, \Sigma s_{i}\right)\right|_{\mathscr{T}}$ are the simple composition factors of $\mathscr{C}(-, \Sigma c) \mid \mathscr{T}$. Consider the following condition on $U \subseteq T$ :

$$
c \in{ }^{\perp}(\Sigma \mathscr{U}) \cap{ }^{\perp}\left(\Sigma^{2} \mathscr{U}\right) \text { and } s_{i} \in \perp \mathscr{U} \cap \perp^{\perp}(\Sigma \mathscr{U}) \cap{ }^{\perp}\left(\Sigma^{2} \mathscr{U}\right) \text { for each } i \text {; }
$$

there are such sets with $T \backslash U$ finite by the first part of Remark [5.4 and as noted, $\mathscr{U}=$ add $U$ is necessarily functorially finite.

We will draw some conclusions about this situation in Lemma 5.5 and Theorem 5.6. Observe that since $c \in^{\perp}(\Sigma \mathscr{U})$, we can view $c$ as an object of both $\mathscr{C}$ and $\mathscr{C}_{U}$.

Consider the (non-commutative) square

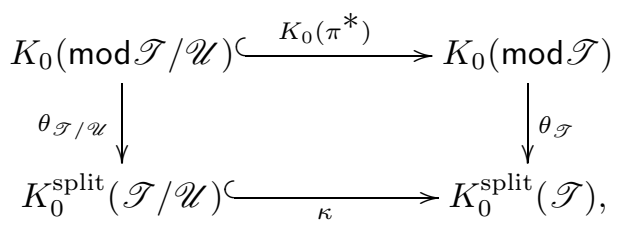

where $\theta_{\mathscr{T}}, \theta_{\mathscr{T} / \mathscr{U}}$ are defined in Paragraph 1.5 and $\kappa$ above. 
Lemma 5.5. Under the above conditions (8), let e be a class in $K_{0}(\bmod \mathscr{T} / \mathscr{U})$ coming from a submodule of $\mathscr{C}_{U}\left(-, \Sigma_{U} c\right) \mid \mathscr{T} / \mathscr{U}$. Then we have

$$
\theta_{\mathscr{T}} \circ K_{0}\left(\pi^{*}\right)(e)=\kappa \circ \theta_{\mathscr{T} / \mathscr{U}}(e) .
$$

Proof. Since the objects $c, s_{1}, \ldots, s_{n}$ belong to the subcategory ${ }^{\perp}(\Sigma \mathscr{U})$, the functors $\left.\mathscr{C}_{U}\left(-, \Sigma_{U} c\right)\right|_{\mathscr{T} / \mathscr{U}},\left.\mathscr{C}_{U}\left(-, \Sigma_{U} s_{i}\right)\right|_{\mathscr{T} / \mathscr{U}}$ are well defined and finitely presented. Moreover, by Paragraph 3.4 their images under $\pi^{*}$ are $\mathscr{C}(-, \Sigma c)\left|\mathscr{T}, \mathscr{C}\left(-, \Sigma s_{i}\right)\right|_{\mathscr{T}}$. It follows from Proposition $3.5\left(\right.$ iii) that the modules $\left.\mathscr{C}_{U}\left(-, \Sigma_{U} s_{i}\right)\right|_{\mathscr{T} / \mathscr{U}}$ are the simple composition factors of $\left.\mathscr{C}_{U}\left(-, \Sigma_{U} c\right)\right|_{\mathscr{T} / \mathscr{U}}$ in $\bmod \mathscr{T} / \mathscr{U}$. Denote by $e_{i}$ the class of $\left.\mathscr{C}_{U}\left(-, \Sigma_{U} s_{i}\right)\right|_{\mathscr{T} / \mathscr{U}}$ in $K_{0}(\bmod \mathscr{T} / \mathscr{U})$. So the class $e$ is a linear combination of the classes $e_{i}$ and it is enough to prove the proposition for the $e_{i}$. However,

$$
K_{0}\left(\pi^{*}\right)\left(e_{i}\right)=\left[\left.\mathscr{C}\left(-, \Sigma s_{i}\right)\right|_{\mathscr{T}}\right],
$$

so

$$
\theta_{\mathscr{T}} \circ K_{0}\left(\pi^{*}\right)\left(e_{i}\right)=\operatorname{coind}_{\mathscr{T}} \Sigma s_{i}-\operatorname{ind}_{\mathscr{T}} \Sigma s_{i} .
$$

On the other hand,

$$
\theta_{\mathscr{T} / \mathscr{U}}\left(e_{i}\right)=\operatorname{coind}_{\mathscr{T} / \mathscr{U}} \Sigma_{U} s_{i}-\operatorname{ind} \mathscr{T}_{\mathscr{U}} \Sigma_{U} s_{i} .
$$

The result thus follows from Lemma 5.2 because $s_{i} \in{ }^{\perp} \mathscr{U} \cap{ }^{\perp}(\Sigma \mathscr{U}) \cap{ }^{\perp}\left(\Sigma^{2} \mathscr{U}\right)$.

Theorem 5.6. Under the above conditions (8), we have

$$
\rho^{\mathscr{T}}(c)=\rho^{\mathscr{T} / \mathscr{U}}(c) .
$$

Proof. By Lemma 5.2 (ii) we have $\operatorname{coind} \mathscr{\mathscr { T }} \Sigma c=\kappa\left(\operatorname{coind} \mathscr{T}_{\mathscr{U}} \Sigma_{U} c\right)$, so we can concentrate on the sums in the Caldero-Chapoton formula.

We know from Paragraph 3.4 that the functor $\pi^{*}$ sends the finitely presented $\mathscr{T} \mid \mathscr{U}$-module $\left.\mathscr{C}_{U}\left(-, \Sigma_{U} c\right)\right|_{\mathscr{T} / \mathscr{U}}$ to the $\mathscr{T}$-module $\left.\mathscr{C}(-, \Sigma c)\right|_{\mathscr{T}}$, and from Proposition 3.5(iii) that it induces a bijection from the isomorphism classes of submodules of $\left.\mathscr{C}_{U}\left(-, \Sigma_{U} c\right)\right|_{\mathscr{T} / \mathscr{U}}$ in $\bmod \mathscr{T} / \mathscr{U}$ to the isomorphism classes of submodules of $\mathscr{C}(-, \Sigma c) \mid \mathscr{T}$ in $\bmod \mathscr{T}$.

Let $e$ be in $K_{0}(\bmod \mathscr{T} / \mathscr{U})$. Then $\pi^{*}$ induces an isomorphism of varieties from $\operatorname{Gr}_{e}\left(\left.\mathscr{C}_{U}\left(-, \Sigma_{U} c\right)\right|_{\mathscr{T} / \mathscr{U}}\right)$ to $\mathrm{Gr}_{K_{0}\left(\pi^{*}\right)(e)}\left(\left.\mathscr{C}(-, \Sigma c)\right|_{\mathscr{T}}\right)$. Moreover, the classes in the Grothendieck group $K_{0}(\bmod \mathscr{T})$ corresponding to non-vanishing terms in $\rho^{\mathscr{T}}(c)$ are all of the form $K_{0}\left(\pi^{*}\right)(e)$, for some $e \in K_{0}(\bmod \mathscr{T} / \mathscr{U})$. Therefore it only remains to be checked that

$$
x^{\theta_{\mathscr{T} / \mathscr{U}}(e)}=x^{\theta_{\mathscr{T}}\left(K_{0}\left(\pi^{*}\right) e\right)}
$$

for elements $e \in K_{0}(\bmod \mathscr{T} / \mathscr{U})$ representing submodules of $\mathscr{C}_{U}\left(-, \Sigma_{U} c\right) \mid \mathscr{T} / \mathscr{U}$. But this holds by Lemma 5.5 .

\section{The Cluster category of Dynkin type $A_{\infty}$}

This section applies the results of the previous sections to $\mathscr{D}$, the cluster category of type $A_{\infty}$ of [16]. It has no cluster tilting objects, but does have a rich supply of cluster tilting subcategories as explained below.

We obtain cluster maps on $\mathscr{D}$, establish some properties, most importantly positivity, and show that some cluster maps cannot be nicely extended to cluster characters. 
6.1 (The cluster category of type $A_{\infty}$ ). This category was studied in [16; we denote it by $\mathscr{D}$. We still let $\mathscr{T}$ denote a cluster tilting subcategory and write $T=\operatorname{ind} \mathscr{T}$. Let us recall some features of this situation.

The results of Sections 11 and 2 apply to $\mathscr{D}$ and $\mathscr{T}$ because Setups 1.1 and 2.1 hold by [16, rmk. 1.2 and thm. 5.2]. In particular, Theorem 2.3 gives a cluster map

$$
\rho:\left.\operatorname{obj} \mathscr{E} \rightarrow \mathbb{Q}\left(x_{t}\right)\right|_{t \in T}
$$

with $\rho(t)=x_{t}$ for $t \in T$. Recall that $\mathscr{E}=\operatorname{add} E$, where $E$ is the set of indecomposable objects of $\mathscr{D}$ which can be reached by finitely many mutations from $\mathscr{T}$.

The AR quiver of $\mathscr{D}$ is $\mathbb{Z} A_{\infty}$ by [16, rmk. 1.4]. The following coordinate system on the quiver is useful.

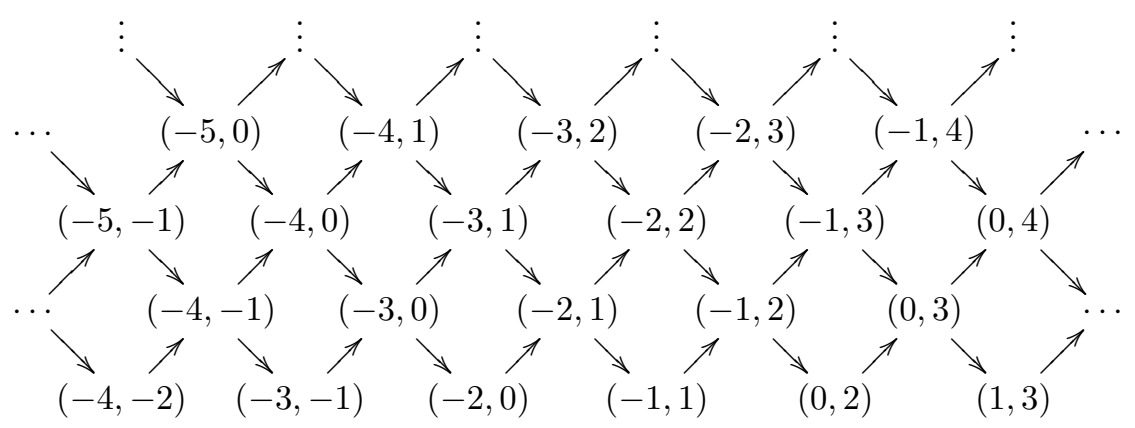

This establishes a bijection between indecomposable objects and $\operatorname{arcs}(m, n)$ connecting non-neighbouring integers. Hence the set $T$ of indecomposable objects corresponds to a collection $\mathfrak{T}$ of arcs.

It was shown in [16, thms. 4.3 and 4.4$]$ that $\mathscr{T}=\operatorname{add} T$ being a cluster tilting subcategory is equivalent to $\mathfrak{T}$ being a maximal collection of non-crossing arcs which is locally finite or has a fountain. Locally finite means that for each integer $n$, only finitely many arcs of the form $(m, n)$ and $(n, p)$ are in $\mathfrak{T}$. Having a fountain means that there is an integer $n$ such that $\mathfrak{T}$ contains infinitely many arcs of the form $(m, n)$ and infinitely many of the form $(n, p)$. The next figures show examples of these two types of $\mathfrak{T}$.
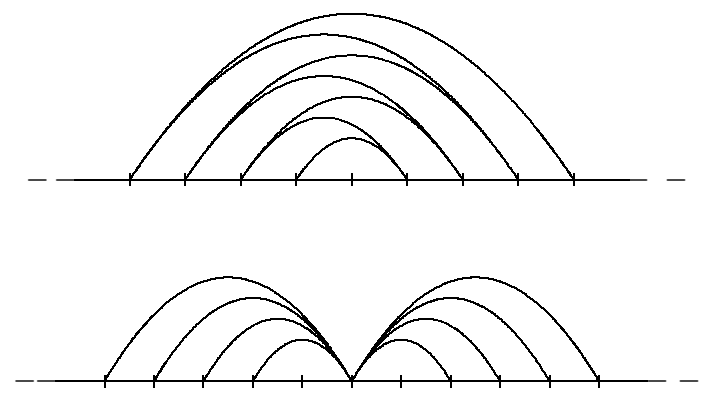

In the case of the fountain, $\mathscr{T}$ contains infinitely many indecomposable objects on each of the diagonal half-lines $(-, n)$ and $(n,-)$ in the AR quiver of $\mathscr{D}$.

We will say that the $\operatorname{arc} \mathfrak{t}=(s, t)$ spans the $\operatorname{arc} \mathfrak{u}=(u, v)$ if $s \leq u<v<t$ or $s<u<v \leq t$. Note that an arc does not span itself. 
Theorem 6.2. Consider the cluster map $\rho:\left.\operatorname{obj} \mathscr{E} \rightarrow \mathbb{Q}\left(x_{t}\right)\right|_{t \in T}$. The subcategory $\mathscr{E}$ is determined as follows:

(i) If $\mathfrak{T}$ is locally finite, then $\mathscr{E}=\mathscr{D}$.

(ii) If $\mathfrak{T}$ has a fountain at $n$, then $\mathscr{E}$ is add of the indecomposable objects which are on or below one of the half-lines $(-, n)$ and $(n,-)$ in the AR quiver of $\mathscr{D}$.

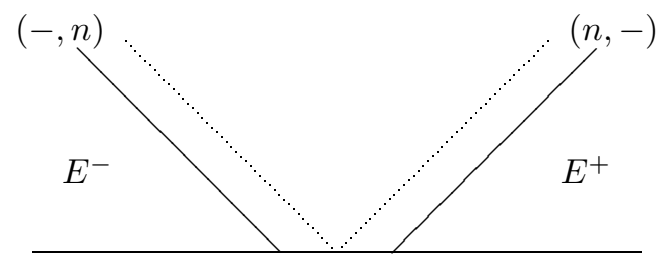

Proof. In (i) we need to see that $\mathscr{E}$ contains all indecomposable objects of $\mathscr{D}$, and in (ii) that it contains precisely the indecomposable objects which are on or below one of the half-lines $(-, n)$ and $(n,-)$.

Each $\mathfrak{t} \in \mathfrak{T}$ divides $\mathfrak{T}$ into two parts: the arcs spanned by $\mathfrak{t}$ and the rest. The arcs in $\mathfrak{T}$ which are spanned by $\mathfrak{t}=(s, t)$ can be viewed as diagonals of a finite polygon $p_{\mathfrak{t}}$ with vertices $\{s, \ldots, t\}$, and as such they form a maximal collection of non-crossing diagonals of $p_{\mathfrak{t}}$, that is, a triangulation of $p_{\mathfrak{t}}$.

(i) Let $d \in \mathscr{D}$ be indecomposable and let $\mathfrak{d}$ be the corresponding arc. It is not difficult to show that since $\mathfrak{T}$ is locally finite, $\mathfrak{d}$ is spanned by an $\operatorname{arc} \mathfrak{t} \in \mathfrak{T}$, so $\mathfrak{d}$ can be viewed as a diagonal of $p_{\mathfrak{t}}$. The arcs in $\mathfrak{T}$ which are spanned by $\mathfrak{t}$ form a triangulation of $p_{\mathfrak{t}}$, and it is clear that by finitely many mutations, this triangulation can be changed into a triangulation containing $\mathfrak{d}$.

But then an equivalent sequence of mutations changes $\mathfrak{T}$ into a maximal collection of non-crossing arcs which contains $\mathfrak{d}$, and accordingly, it changes $\mathscr{T}$ into a cluster tilting subcategory containing $d$. So $d \in \mathscr{E}$.

(ii) Let $d \in \mathscr{D}$ be indecomposable and let $\mathfrak{d}=(p, q)$ be the corresponding arc. If $d$ is on or below one of the half-lines $(-, n)$ and $(n,-)$, then either $p<q \leq n$ or $n \leq p<q$; assume the former for the sake of argument. Since $\mathfrak{T}$ has a fountain at $n$, it follows that there is a $\mathfrak{t}=(m, n)$ in $\mathfrak{T}$ which spans $\mathfrak{d}$. Now proceed as in part (i) to see that $d \in \mathscr{E}$.

If $d$ is above the half-lines $(-, n)$ and $(n,-)$, then $p<n<q$. This means that $\mathfrak{d}$ crosses an infinite number of arcs in $\mathfrak{T}$, so there is no finite sequence of mutations which changes $\mathfrak{T}$ into a maximal collection of non-crossing arcs which contains $\mathfrak{d}$, and accordingly, no finite sequence of mutations which changes $\mathscr{T}$ into a cluster tilting subcategory containing $d$. Hence $d \notin \mathscr{E}$.

Proposition 6.3. Suppose that $\mathfrak{T}$ has a fountain at $n$. Consider the sets $E^{-}$and $E^{+}$of indecomposable objects of $\mathscr{D}$ defined by the figure in Theorem 6.2(ii).

If $d \in E^{-}$, then $\rho(d)$ can be written using variables $x_{t}$ with $t \in T \cap E^{-}$, and if $d \in E^{+}$, then $\rho(d)$ can be written using variables $x_{t}$ with $t \in T \cap E^{+}$.

Proof. For the sake of argument, suppose $d \in E^{-}$and let $\mathfrak{d}$ be the corresponding arc.

As in the proof of Theorem 6.2(ii), there is a $\mathfrak{t}=(m, n)$ in $\mathfrak{T}$ which spans $\mathfrak{d}$. As in the proof of Theorem 6.2(i), the arcs in $\mathfrak{T}$ which are spanned by $\mathfrak{t}$ can be viewed as a triangulation of the polygon $p_{\mathfrak{t}}$ with vertices $\{m, \ldots, n\}$, and by finitely many 
mutations, this triangulation can be changed into a triangulation containing $\mathfrak{d}$. The $\operatorname{arcs} \mathfrak{c}_{1}, \ldots, \mathfrak{c}_{k}=\mathfrak{d}$ involved in the mutations are spanned by $\mathfrak{t}$.

An equivalent sequence of mutations changes $\mathfrak{T}$ into a maximal collection of non-crossing arcs which contains $\mathfrak{d}$, and accordingly, it changes $\mathscr{T}$ into a cluster tilting subcategory containing $d$. Since the $\mathfrak{c}_{i}$ are spanned by $\mathfrak{t}$, the corresponding indecomposable objects $c_{1}, \ldots, c_{k}=d$ involved in the mutations are in $E^{-}$.

However, add $E^{-}$is closed under extensions since it is equal to $R^{\perp}$, where $R$ consists of the indecomposable objects on the half-lines $(n+1,-)$ and $(n+2,-)$. Hence the middle terms of the exchange triangles $c_{i} \rightarrow b_{i} \rightarrow c_{i+1}$ and $c_{i+1} \rightarrow b_{i}^{\prime} \rightarrow c_{i}$ are direct sums of indecomposable objects from $E^{-}$. Applying Property 2.2(iii) of cluster maps successively to these triangles expresses $\rho(d)$ in terms of variables $x_{t}$ with $t \in E^{-}$.

Definition 6.4. For $t \in T$, let $\mathfrak{t} \in \mathfrak{T}$ be the corresponding arc under the bijection of Paragraph 6.1 and set

$$
\mathfrak{U}(\mathfrak{t})=\{\mathfrak{u} \in \mathfrak{T} \mid \text { the } \operatorname{arc} \mathfrak{u} \text { is not spanned by the } \operatorname{arc} \mathfrak{t}\} \subseteq \mathfrak{T} .
$$

The bijection described in Paragraph 6.1 says that $\mathfrak{U}(\mathfrak{t})$ corresponds to a set of indecomposable objects $U(t) \subseteq T$. Setting $\mathscr{U}(t)=$ add $U(t)$ gives a subcategory $\mathscr{U}(t) \subseteq \mathscr{T}$.

Note that $\mathfrak{t} \in \mathfrak{U}(\mathfrak{t})$, so $t \in \mathscr{U}(t)$. Moreover, there are only finitely many arcs spanned by $\mathfrak{t}$, so $\mathfrak{U}(\mathfrak{t})$ consists of all but finitely many of the arcs in $\mathfrak{T}$, and hence $U(t)$ consists of all but finitely many of the indecomposable objects in $T$.

Lemma 6.5. The subcategory $\mathscr{U}(t)$ is functorially finite in $\mathscr{D}$, and the Calabi-Yau reduction $\mathscr{D}_{U(t)}$ is a cluster category of Dynkin type $A_{m}$, where $m$ is the number of arcs in $\mathfrak{T}$ spanned by $\mathfrak{t}$.

Proof. Since $U(t)$ consists of all but finitely many of the indecomposable objects in $T$, it follows from Lemma 4.1 that $\mathscr{U}(t)$ is functorially finite in $\mathscr{D}$.

The Calabi-Yau reduction $\mathscr{D}_{U(t)}={ }^{\perp}(\Sigma \mathscr{U}(t)) /[\mathscr{U}(t)]$ is a triangulated Homfinite 2-Calabi-Yau category. Since a Calabi-Yau reduction of a 2-Calabi-Yau algebraic category is again algebraic [1, Prop 4.3], the category $\mathscr{D}_{U(t)}$ is algebraic. To see that it is a cluster category of type $A_{m}$, by [20, secs. 2 and 4$]$ it is sufficient to show that it has a cluster tilting object $V$ with $\operatorname{End}(V) \cong k A_{m}$.

By the theory of Calabi-Yau reductions, as explained in Paragraph 3.2 the cluster tilting subcategories of $\mathscr{D}_{U(t)}$ have the form $\mathscr{V} / \mathscr{U}(t)$, where $\mathscr{V}$ is a cluster tilting subcategory of $\mathscr{D}$ with $\mathscr{U}(t) \subseteq \mathscr{V}$. Such a $\mathscr{V}$ corresponds to a maximal collection $\mathfrak{V}$ of non-crossing $\operatorname{arcs}$ with $\mathfrak{U}(\mathfrak{t}) \subseteq \mathfrak{V}$. Let us construct a set $\mathfrak{V}$ by starting with $\mathfrak{U}(\mathfrak{t})$ and adding the following arcs spanned by $\mathfrak{t}$.

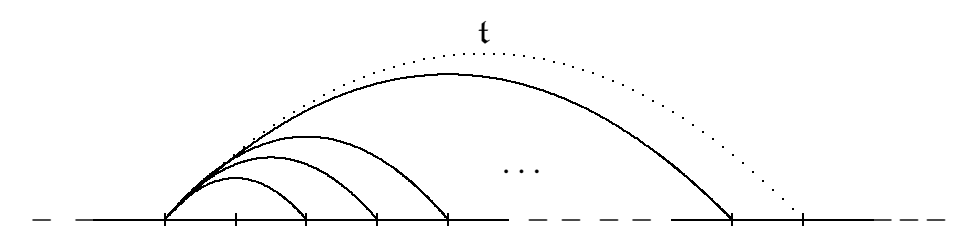


Then the indecomposable objects of $\mathscr{V}$ are those of $\mathscr{U}(t)$ along with finitely many indecomposable objects placed as follows on a line segment below $t$ in the AR quiver of $\mathscr{D}$.

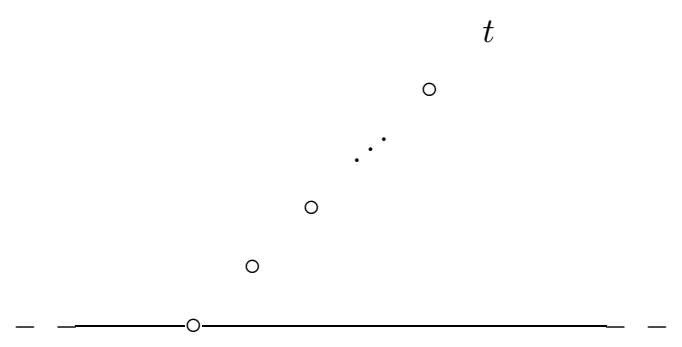

The cluster tilting subcategory $\mathscr{V} / \mathscr{U}(t)$ of $\mathscr{D}_{U(t)}$ has only finitely many indecomposable objects given by the indecomposable objects marked by $\circ$ in the figure. Their direct sum gives a cluster tilting object $V$ of $\mathscr{D}_{U(t)}$, and if there are $m$ of them, then it is not hard to show that $\operatorname{End}(V) \cong k A_{m}$. Here $m$ is the number of arcs in a triangulation of the polygon $p_{\mathfrak{t}}$, that is, the number of arcs in $\mathfrak{T}$ spanned by $\mathfrak{t}$.

Remark 6.6. The authors believe that all the Calabi-Yau reductions of $\mathscr{D}$ are products of cluster categories of type $A$. If one had a detailed description of rigid subcategories of the category $\mathscr{D}$, then this could be proven by using Lemma 4.1 and the main theorem of [20. Another possible approach might be to use the geometric description of the cluster category of type $A_{m}$ due to [11.

Lemma 6.7. Let $d \in \mathscr{E}$ be an indecomposable object. There exists $t \in T$ such that $d \in{ }^{\perp}(\Sigma \mathscr{U}(t)) \backslash \mathscr{U}(t)$ and $\left.\rho(d) \in \mathbb{Q}\left(x_{t^{\prime}}\right)\right|_{t^{\prime} \in T \backslash U(t)}$.

Proof. Let $T^{\prime}$ be a finite subset of $T$ such that $\rho(d)$ can be written in terms of the $x_{t^{\prime}}$ for $t^{\prime} \in T^{\prime}$. We must show that there is a $t \in T$ such that $d \in{ }^{\perp}(\Sigma \mathscr{U}(t)) \backslash \mathscr{U}(t)$ and $T^{\prime} \subseteq T \backslash U(t)$. Since $\mathscr{U}(t)=$ add $U(t)$, it is enough to show that there is a $t \in T$ such that $d$ and $T^{\prime}$ are both contained in ${ }^{\perp}(\Sigma \mathscr{U}(t)) \backslash \mathscr{U}(t)$.

Let us rephrase this in terms of arcs. Let $d$ correspond to the $\operatorname{arc} \mathfrak{d}$ and $T^{\prime}$ to the finite collection $\mathfrak{T}^{\prime} \subset \mathfrak{T}$. We must show that there is a $\mathfrak{t} \in \mathfrak{T}$ such that $\mathfrak{d}$ and $\mathfrak{T}^{\prime}$ are both contained in the set $\mathfrak{P}(\mathfrak{t})$ of arcs corresponding to the indecomposable objects of ${ }^{\perp}(\Sigma \mathscr{U}(t)) \backslash \mathscr{U}(t)$. Let us show that

$$
\mathfrak{P}(\mathfrak{t})=\{\mathfrak{p} \text { is an } \operatorname{arc} \mid \mathfrak{p} \text { is spanned by } \mathfrak{t}\} .
$$

Namely, [16, lem. 3.6] shows that the indecomposable objects in ${ }^{\perp}(\Sigma \mathscr{U}(t))$ correspond to the arcs which do not cross any arc in $\mathfrak{U}(\mathfrak{t})$. So the indecomposable objects in ${ }^{\perp}(\Sigma \mathscr{U}(t)) \backslash \mathscr{U}(t)$ correspond to the arcs which do not cross any arc in $\mathfrak{U}(\mathfrak{t})$ and are outside $\mathfrak{U}(\mathfrak{t})$. Combining this with the definition of $\mathfrak{U}(\mathfrak{t})$ shows equality (10).

Now, if $\mathfrak{T}$ is locally finite, then it is easy to show that, given any finite collection $\mathfrak{Q}$ of arcs, there is a $\mathfrak{t} \in \mathfrak{T}$ such that $\mathfrak{Q} \subseteq \mathfrak{P}(\mathfrak{t})$.

If $\mathfrak{T}$ has a fountain at $n$, then Theorem 6.2 (ii) implies that the indecomposable object $d$ is in either $E^{-}$or $E^{+}$. For the sake of argument, suppose $d \in E^{-}$. Then $T^{\prime}$ can be chosen as a subset of $E^{-}$by Proposition 6.3. But then $\mathfrak{d}$ and each $\mathfrak{t}^{\prime} \in \mathfrak{T}^{\prime}$ is an arc of the form $(p, q)$ with $p<q \leq n$. Since $\mathfrak{T}$ has a fountain at $n$, it follows that there is a $\mathfrak{t}=(m, n)$ in $\mathfrak{T}$ which spans $\mathfrak{d}$ and each $\mathfrak{t}^{\prime}$, so $\mathfrak{d} \in \mathfrak{P}(\mathfrak{t})$ and $\mathfrak{T}^{\prime} \subseteq \mathfrak{P}(\mathfrak{t})$ as desired. 
Theorem 6.8. The cluster map $\rho^{\mathscr{T}}: \operatorname{obj} \mathscr{E} \rightarrow \mathbb{Q}\left(x_{t}\right)_{t \in T}$ enjoys the following properties.

(i) $\rho^{\mathscr{T}}(t)=x_{t}$ for $t \in T$.

(ii) If $d \in \mathscr{E}$, then $\rho^{\mathscr{T}}(d)$ is a non-zero Laurent polynomial.

(iii) In each such Laurent polynomial, the coefficients in the numerator are positive integers.

Proof. Part (i) is already in Theorem 2.3. In (ii) it is clear from the definition in Paragraph 1.8 that $\rho^{\mathscr{T}}(d)$ is a Laurent polynomial.

For the rest of the proof, we can assume that $d \in \mathscr{E}$ is indecomposable. Use Lemma 6.7 to pick $t \in T$ such that $d \in{ }^{\perp}(\Sigma \mathscr{U}(t)) \backslash \mathscr{U}(t)$ and

$$
\left.\rho^{\mathscr{T}}(d) \in \mathbb{Q}\left(x_{t^{\prime}}\right)\right|_{t^{\prime} \in T \backslash U(t)} .
$$

The Calabi-Yau reduction $\mathscr{D}_{U(t)}$ is a cluster category of type $A_{m}$ by Lemma 6.5. so viewed in $\mathscr{D}_{U(t)}$ the object $d$ is reachable from the cluster tilting subcategory $\mathscr{T} / \mathscr{U}(t)$. Hence Theorem 4.8 gives $\left.\rho^{\mathscr{T}}(d)\right|_{x_{u}=1 \text { for } u \in U(t)}=\rho^{\mathscr{T} / \mathscr{U}(t)}(d)$, and by equation (11) this implies that $\rho^{\mathscr{T}}(d)=\rho^{\mathscr{T} / \mathscr{U}(t)}(d)$. But it follows from [24, thm. 4] or Theorem 2.3 that $\rho^{\mathscr{T} / \mathscr{U}(t)}$ is a cluster map on $\mathscr{D}_{U(t)}$, and the remaining parts of (ii) and (iii) now follow from [12, thm. 3] or [27, cor. 3.6].

Remark 6.9. By Theorem 6.2(ii), when $\mathscr{T}$ corresponds to a fountain, $\mathscr{E}$ is not all of $\mathscr{D}$. It is reasonable to ask in this case whether $\rho$ can be extended to all of $\mathscr{D}$. Let us show that the answer is no in the case of the simplest fountain given by figure (9) of Paragraph 6.1.

Theorem 6.10. Suppose that $\mathscr{T}$ corresponds to the fountain (9). Then there is no cluster map $\rho: \operatorname{obj} \mathscr{D} \rightarrow \mathbb{Q}\left(x_{t}\right)_{t \in T}$ which satisfies the conditions of Theorem 6.8.

Proof. Suppose that such a $\rho$ exists. By setting all the $x_{t}$ equal to 1 we obtain a map $\psi: \operatorname{obj} \mathscr{D} \rightarrow\{1,2,3, \ldots\}$.

Moreover, $T$ consists of the indecomposable objects on the half-lines $(-, n)$ and $(n,-)$ sketched in Theorem 6.2. Let us consider the indecomposables $t_{i}$ on $(-, n)$ and $u_{i}$ on the half-line above it.

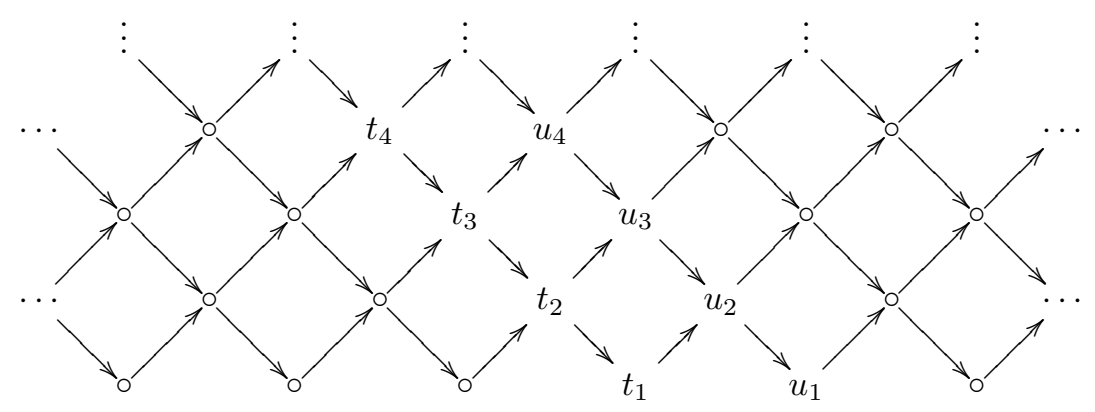

We have $\operatorname{dim}_{k} \operatorname{Ext}^{1}\left(u_{i}, t_{i}\right)=1$ for each $i$. For $i=1$ there is an AR triangle $t_{1} \rightarrow u_{2} \rightarrow u_{1}$ and a non-split distinguished triangle $u_{1} \rightarrow 0 \rightarrow t_{1}$; note that the connecting morphism of this triangle is the isomorphism $t_{1} \rightarrow \Sigma u_{1}$. The defining properties of cluster maps now imply that $\rho$ satisfies

$$
\rho\left(t_{1}\right) \rho\left(u_{1}\right)=\rho\left(u_{2}\right)+1 \text {. }
$$


Likewise, for each $i \geq 2$ there is an AR triangle $t_{i} \rightarrow t_{i-1} \oplus u_{i+1} \rightarrow u_{i}$ and a nonsplit distinguished triangle $u_{i} \rightarrow 0 \rightarrow t_{i}$; the connecting morphism of this triangle is the isomorphism $t_{i} \rightarrow \Sigma u_{i}$. Therefore $\rho$ satisfies

$$
\rho\left(t_{i}\right) \rho\left(u_{i}\right)=\rho\left(t_{i-1}\right) \rho\left(u_{i+1}\right)+1 \text { for } i \geq 2 .
$$

The displayed equations can also be written as $x_{t_{1}} \rho\left(u_{1}\right)=\rho\left(u_{2}\right)+1$ and $x_{t_{i}} \rho\left(u_{i}\right)$ $=x_{t_{i-1}} \rho\left(u_{i+1}\right)+1$ for $i \geq 2$. Setting all the $x_{t}$ equal to 1 , this implies that $\psi$ satisfies $\psi\left(u_{i}\right)=\psi\left(u_{i+1}\right)+1$ for each $i \geq 1$. But this contradicts that the values of $\psi$ are positive.

\section{7. $\mathrm{SL}_{2}$-TILINGS}

This section has an application of our results to $\mathrm{SL}_{2}$-tilings as introduced in $[3]$. Similar techniques appeared recently in [2].

Definition 7.1. The following notion was introduced in [3]: An $\mathrm{SL}_{2}$-tiling of $\mathbb{Z}^{2}$ with values in a commutative ring $R$ is a map $r: \mathbb{Z} \times \mathbb{Z} \rightarrow R$ for which each matrix

$$
\left(\begin{array}{cc}
r(i, j) & r(i, j+1) \\
r(i+1, j) & r(i+1, j+1)
\end{array}\right)
$$

has determinant 1 . That is,

$$
r(i, j) r(i+1, j+1)-r(i, j+1) r(i+1, j)=1 .
$$

We consider a modified version of this. An $\mathrm{SL}_{2}$-tiling of the half-plane $Q=$ $\{(m, n) \mid m \leq n-2\} \subset \mathbb{Z}^{2}$ is a map $r: Q \rightarrow R$ for which the above matrix has determinant 1 when it makes sense. Along the edge of $Q$ the matrix does not make sense, but here we require that

$$
\left(\begin{array}{cc}
r(i, i+2) & r(i, i+3) \\
1 & r(i+1, i+3)
\end{array}\right)
$$

has determinant 1 for each $i$. That is,

$$
r(i, i+2) r(i+1, i+3)-r(i, i+3)=1 .
$$

Remark 7.2. Note that $Q$ consists precisely of the coordinate pairs in the coordinate system on the AR quiver of $\mathscr{D}$ given in Paragraph 6.1. We can view the elements of $Q$ as $\operatorname{arcs}(m, n)$ connecting non-neighbouring integers, or as indecomposable objects of $\mathscr{D}$.

The following result shows that certain patterns of 1's distributed in $Q$ can be extended to $\mathrm{SL}_{2}$-tilings.

Theorem 7.3. Let $\mathfrak{T}$ be a locally finite maximal collection of non-crossing arcs. Then there is an $\mathrm{SL}_{2}$-tiling $r: Q \rightarrow\{1,2,3, \ldots\}$ which satisfies $r(\mathfrak{t})=1$ for each $\mathfrak{t} \in \mathfrak{T}$.

Proof. By Paragraph 6.1, the collection $\mathfrak{T}$ corresponds to the indecomposable objects $T$ of a cluster tilting subcategory $\mathscr{T}$ of $\mathscr{D}$, the cluster category of type $A_{\infty}$. We must show that there is an $r$ which satisfies $r(t)=1$ for each $t \in T$.

Theorems 2.3 and 6.2 say that $\mathscr{T}$ gives rise to a cluster map $\rho:$ obj $\mathscr{D} \rightarrow$ $\mathbb{Q}\left(x_{t}\right)_{t \in T}$. By manipulations with AR triangles such as the ones in the proof of Theorem 6.10, ones proves that if $(i, i+2)$ is on the edge of $Q$, then

$$
\rho(i, i+2) \rho(i+1, i+3)=\rho(i, i+3)+1 .
$$


Likewise, if $(i, j) \in Q$ has $i \leq j-3$, then

$$
\rho(i, j) \rho(i+1, j+1)=\rho(i, j+1) \rho(i+1, j)+1 .
$$

Hence the map $\rho$, restricted to elements of $Q$, satisfies equations (12) and (13).

So the map $r$ obtained by setting each $x_{t}$ equal to 1 also satisfies equations (12) and (13). Since $\rho(t)=x_{t}$ for $t \in T$ by Theorem [2.3, it follows that $r(t)=1$ for $t \in T$, and the map $r$ takes values in $\{1,2,3, \ldots\}$ since each $\rho(i, j)$ is a non-zero Laurent polynomial whose numerator is a linear combination of monomials with positive integer coefficients by Theorem 6.8(ii and iii).

Remark 7.4. The main result on $\mathrm{SL}_{2}$-tilings in 3 is that if $S$ is an admissible frontier of 1's in $\mathbb{Z}^{2}$, then $S$ can be extended uniquely to an $\mathrm{SL}_{2}$-tiling $p$ of $\mathbb{Z}^{2}$.

An admissible frontier consists of the coordinate pairs on a doubly infinite path which is built out of the steps $(1,0)$ and $(0,1)$, with the stipulation that the steps must alternate infinitely often in both directions.

Half of a frontier $S$ falls in the half-plane $Q$, and it is not hard to show that the "zig-zag" $T=S \cap Q$ consists of the indecomposable objects of a locally bounded cluster tilting subcategory $\mathscr{T}$ of $\mathscr{D}$. Let us show a sketch of an example.

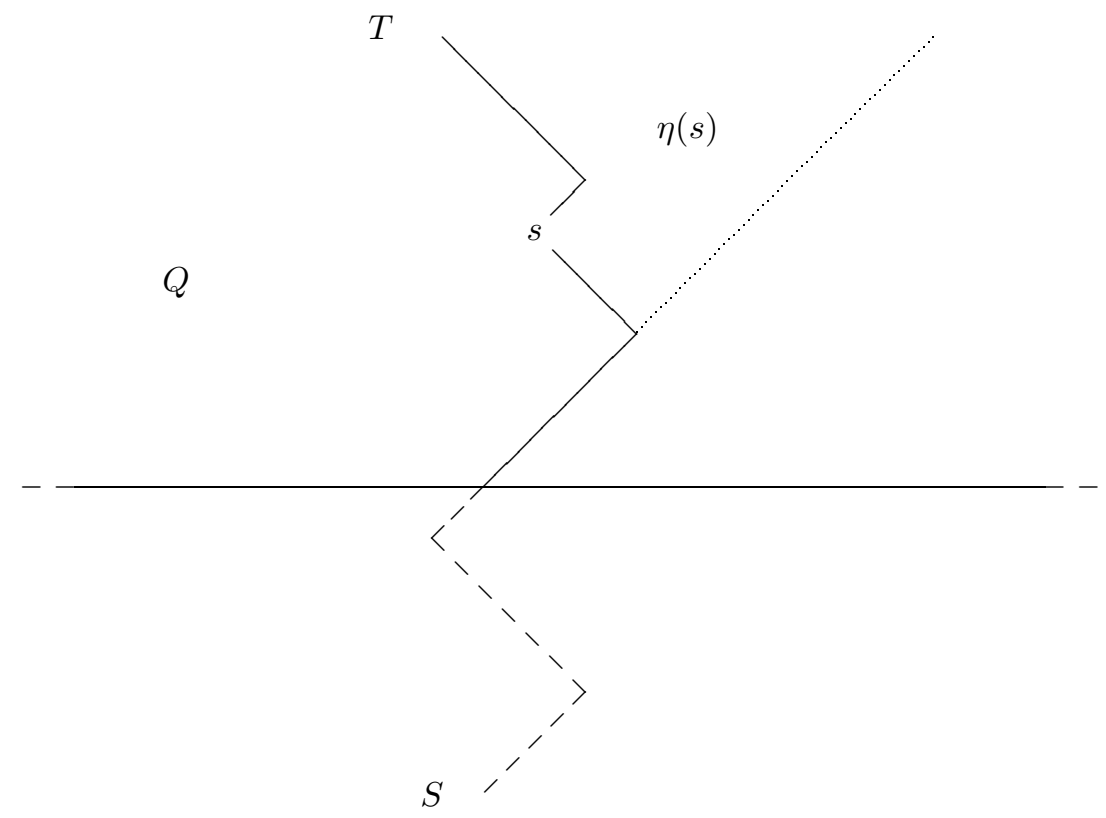

By the correspondence between $T$ 's and T's, Theorem 7.3 gives an $\mathrm{SL}_{2}$-tiling $r$ of $Q$ with $r(t)=1$ for $t \in T$.

Now, starting from a "turning point" $s$ and using that $p$ and $r$ are 1 on $T$, equation (12) alone determines $p$ and $r$ in the region $\eta(s)$ indicated in the sketch. So $p$ and $r$ agree on $\eta(s)$. Moreover, the regions $\eta(s)$ cover $\mathbb{Z}^{2}$ when $s$ ranges over the turning points of $S$, and for a given $s$, it is always possible to move $S$ within $\mathbb{Z}^{2}$ such that $s$ and $\eta(s)$ fall inside $Q$. In this sense, it is possible to obtain the tiling $p$ by gluing different tilings $r$.

However, the "zig-zags" $T=S \cap Q$ correspond to very special examples of locally bounded cluster tilting subcategories. The corresponding locally finite maximal collections of non-crossing arcs are built in the following way, with neighbouring 
arcs going in one direction from $a$, neighbouring arcs going in the opposite direction from $b$, etc.

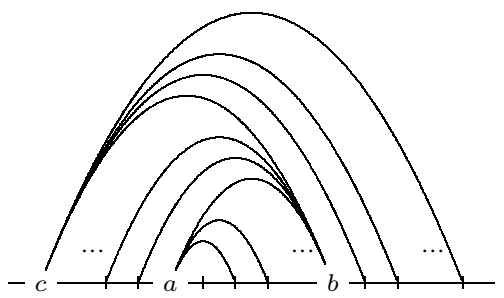

It is clear, for instance by mutating a configuration such as this, that much more general $T$ 's are possible, and Theorem 7.3 also works for these.

It would be interesting to obtain a version of Theorem 7.3 with $\mathbb{Z}^{2}$ instead of $Q$.

\section{ACKNOWLEDGEMENT}

We thank Bernhard Keller for suggesting that the Caldero-Chapoton map can be made to work in the context of cluster tilting subcategories with infinitely many indecomposable objects.

This enabled us to improve the results in a previous version of the paper which used Calabi-Yau reduction to go down to subquotient categories with cluster tilting objects, and "bootstrapping" to lift their cluster characters to the original category. We are grateful to Osamu Iyama, Ralf Schiffler, Dong Yang, and Andrei Zelevinsky for comments to the previous version.

We thank Idun Reiten for answering a question on [6].

The second-named author would like to thank Robert Marsh and the EPSRC for a post-doctoral position in Leeds. He would also like to thank Raf Bocklandt for an invitation to Newcastle during which part of this work was carried out.

\section{REFERENCES}

[1] C. Amiot and S. Oppermann, Cluster equivalence and graded derived equivalence, preprint (2010). arXiv:math.RT/1003.4916.

[2] I. Assem and G. Dupont, Friezes and a construction of the euclidean cluster variables, J. Pure Appl. Algebra 215 (2011), 2322-2340. MR.2793939

[3] I. Assem, C. Reutenauer, and D. Smith, Frises, Adv. Math. 225 (2010), 3134-3165. MR2729004

[4] M. Auslander, "Representation dimension of Artin algebras", Queen Mary College Mathematics Notes, Queen Mary College, London, 1971. Reprinted pp. 505-574 in "Selected works of Maurice Auslander", Vol. 1 (edited by Reiten, Smalø, and Solberg), American Mathematical Society, Providence, RI, 1999. MR1674397 (2000j:01119a)

[5] M. Auslander, Representation theory of Artin algebras II, Comm. Algebra 1 (1974), 269-310. MR0349747 (50:2240)

[6] A. B. Buan, O. Iyama, I. Reiten, and J. Scott, Cluster structures for 2-Calabi-Yau categories and unipotent groups, Compositio Math. 145 (2009), 1035-1079. MR2521253(2010h:18021)

[7] A. B. Buan, R. J. Marsh, M. Reineke, I. Reiten, and G. Todorov, Tilting theory and cluster combinatorics, Adv. Math. 204 (2006), 572-618. MR.2249625(2007f:16033)

[8] A. B. Buan, R. J. Marsh, and I. Reiten, Cluster-tilted algebras, Trans. Amer. Math. Soc. 359 (2007), 323-332. MR2247893 (2007f:16035)

[9] A. B. Buan, R. J. Marsh, I. Reiten, and G. Todorov, Clusters and seeds in acyclic cluster algebras, with an appendix coauthored in addition by P. Caldero and B. Keller, Proc. Amer. Math. Soc. 135 (2007), 3049-3060. MR2322734(2008j:16044)

[10] P. Caldero and F. Chapoton, Cluster algebras as Hall algebras of quiver representations, Comment. Math. Helv. 81 (2006), 595-616. MR2250855 (2008b:16015) 
[11] P. Caldero, F. Chapoton, and R. Schiffler, Quivers with relations arising from clusters $\left(A_{n}\right.$ case), Trans. Amer. Math. Soc. 358 (2006), 1347-1364. MR2187656 (2007a:16025)

[12] P. Caldero and B. Keller, From triangulated categories to cluster algebras, Invent. Math. 172 (2008), 169-211. MR2385670 (2009f:16027)

[13] P. Caldero and B. Keller, From triangulated categories to cluster algebras II, Ann. Sci. École Norm. Sup. (4) 39 (6) (2006), 983-1009. MR2316979 (2008m:16031)

[14] R. Dehy and B. Keller, On the combinatorics of rigid objects in 2-Calabi-Yau categories, Int. Math. Res. Not. IMRN 2008, no. 11, Art. ID rnn029, 17 pages. MR2428855 (2009e:18020)

[15] S. Fomin and A. Zelevinsky, Cluster algebras I. Foundations, J. Amer. Math. Soc. 15 (2002), no. 2, 497-529. MR 1887642 (2003f:16050)

[16] T. Holm and P. Jørgensen, On a cluster category of infinite Dynkin type, and the relation to triangulations of the infinity-gon, Math. Z. 270 (2012), 277-295. MR2875834

[17] O. Iyama and Y. Yoshino, Mutation in triangulated categories and rigid Cohen-Macaulay modules, Invent. Math. 172 (2008), 117-168. MR.2385669 (2008k:16028)

[18] B. Keller, Categorification of acyclic cluster algebras: an introduction, arXiv:0801.3103v1 [math.RT]

[19] B. Keller, Cluster algebras, quiver representations and triangulated categories, pp. 76-160 in "Triangulated Categories" (edited by Holm, Jørgensen, and Rouquier), London Math. Soc. Lecture Note Ser., 375, Cambridge Univ. Press, 2010. MR2681708 (2011h:13033)

[20] B. Keller and I. Reiten, Acyclic Calabi-Yau categories. With an appendix by Michel Van den Bergh, Compositio Math. 144 (2008), 1332-1348. MR2457529 (2009i:18007)

[21] B. Keller and I. Reiten, Cluster tilted algebras are Gorenstein and stably Calabi-Yau, Adv. Math. 211 (2007), 123-151. MR2313531 (2008b:18018)

[22] S. König and B. Zhu, From triangulated categories to abelian categories - cluster tilting in a general framework, Math. Z. 258 (2008), 143-160. MR2350040 (2008m:18021)

[23] H. Lenzing and I. Reiten, Hereditary Noetherian categories of positive Euler characteristic, Math. Z. 254 (2006), 133-171. MR2232010 (2007e:18008)

[24] Y. Palu, Cluster characters for 2-Calabi-Yau triangulated categories, Ann. Inst. Fourier (Grenoble) 58 (2008), 2221-2248. MR2473635 (2009k:18013)

[25] Y. Palu, Cluster characters II: A multiplication formula, Proc. London Math. Soc. 104 (2012), 57-78. MR2876964

[26] P.-G. Plamondon, Cluster characters for cluster categories with infinite-dimensional morphism spaces, Adv. Math. 227 (2011), 1-39. MR2782186

[27] R. Schiffler and H. Thomas, On cluster algebras arising from unpunctured surfaces, Int. Math. Res. Not. IMRN 2009, no. 17, Art. ID rnp047, 30 pages. MR2534994 (2010h:13040)

[28] A. Zelevinsky, What is a cluster algebra?, Notices Amer. Math. Soc. 54 (2007), 1494-1495. MR2361161

School of Mathematics and Statistics, Newcastle University, NewCastle upon Tyne NE1 7RU, United KINGDOM

E-mail address: peter.jorgensen@ncl.ac.uk

URL: http://www.staff.ncl.ac.uk/peter.jorgensen

Department of Pure Mathematics, University of Leeds, Leeds LS2 9JT, United KingDOM

E-mail address: ypalu@maths.leeds.ac.uk

URL: http://www.amsta.leeds.ac.uk/ ypalu

Current address: LAMFA, Université de Picardie Jules Verne, 33, Rue Saint-Leu, 80039 Amiens, France

E-mail address: yann.palu@u-picardie.fr 